\title{
Bringing the \\ ATLAS Muon Spectrometer to Life with Cosmic Rays
}

\author{
D. Orestano \\ Università Roma Tre \& INFN \\ On behalf of the ATLAS Muon Collaboration
}




\section{Cosmic ray event in ATLAS}

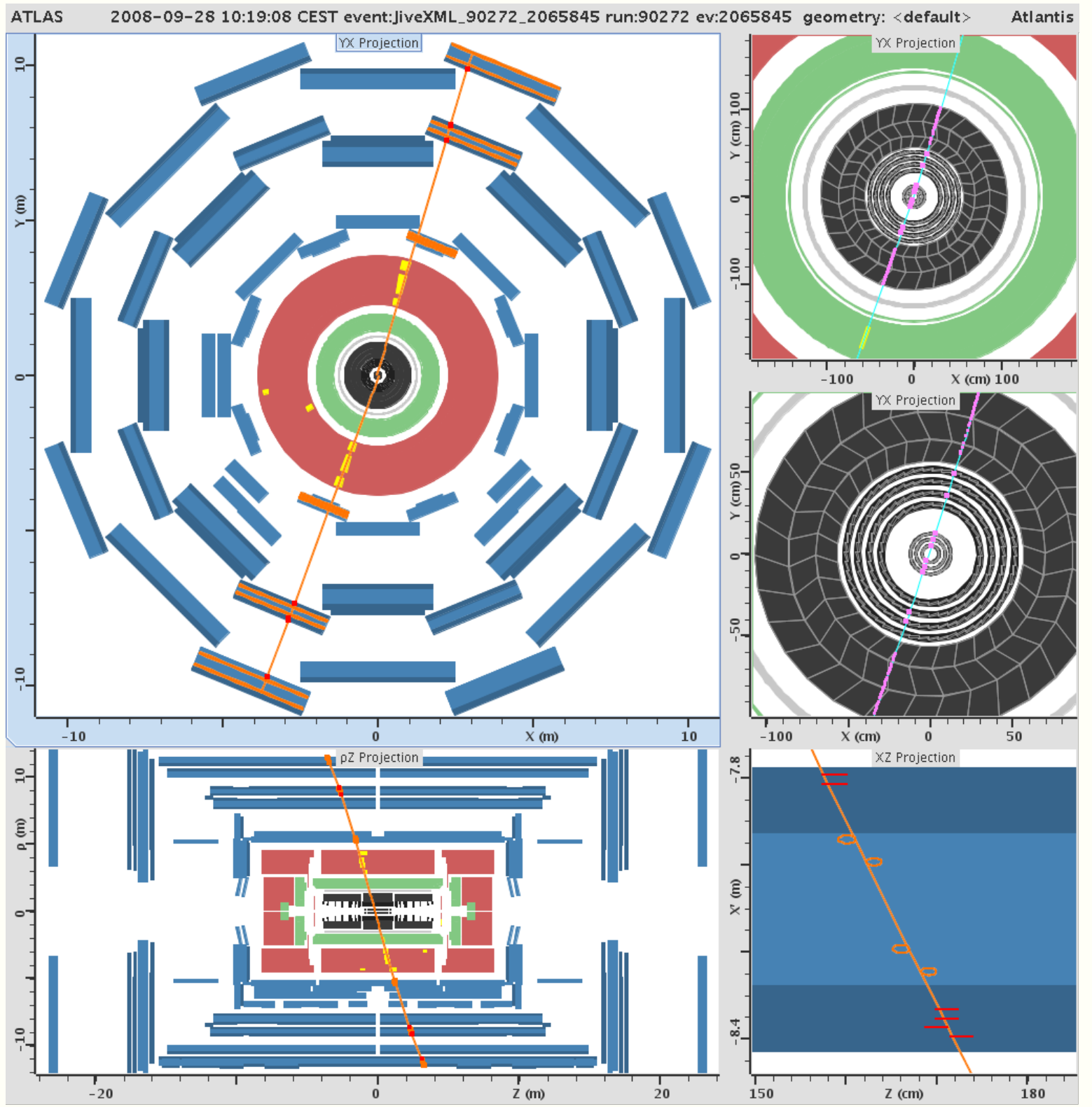




\section{The ATLAS Muon Spectrometer}

\begin{tabular}{|l|c|}
\hline Monitored drift tubes & MDT \\
- Coverage & $|\eta|<2.7$ (innermost layer: $|\eta|<2.0)$ \\
- Number of chambers & 1088 \\
- Number of channels & 339000 \\
- Function & Precision tracking \\
\hline Cathode strip chambers & CSC \\
- Coverage & $2.0<|\eta|<2.7$ \\
- Number of chambers & 32 \\
- Number of channels & 31000 \\
- Function & Precision tracking \\
\hline Resistive plate chambers & RPC \\
- Coverage & $|\eta|<1.05$ \\
- Number of chambers & 544 \\
- Number of channels & 359000 \\
- Function & Triggering, second coordinate \\
\hline Thin gap chambers & TGC \\
- Coverage & $1.05<|\eta|<2.7(2.4$ for triggering) \\
- Number of chambers & 3588 \\
- Number of channels & 318000 \\
- Function & Triggering, second coordinate \\
\hline
\end{tabular}

A complex system:

- 4 different technologies (MDT,CSC,RPC,TGC)

- Large area $\left(10,000 \mathrm{~m}^{2}\right)$

- Many channels (1M)

- Many degrees of freedom (5300 chambers)

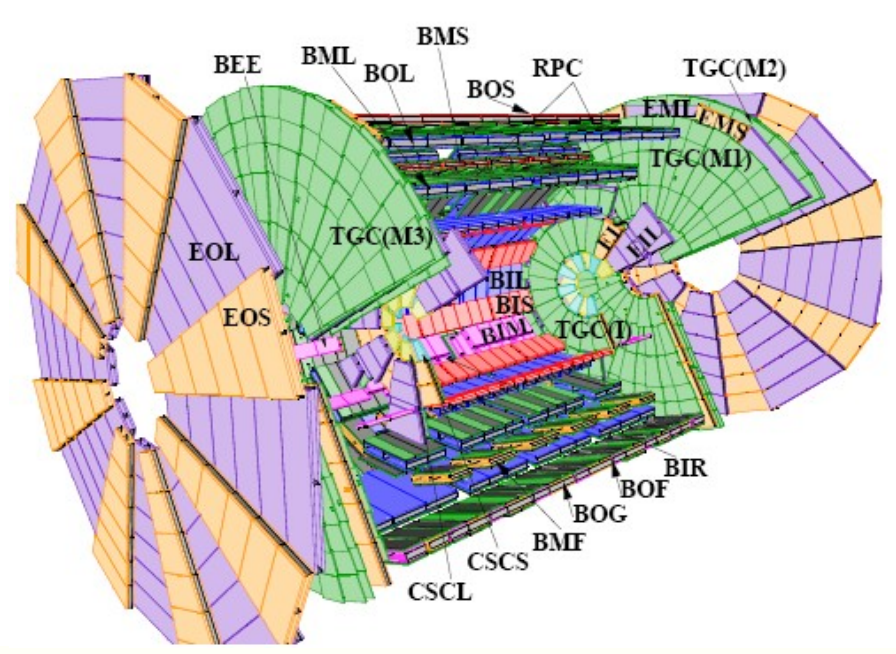




\section{The ATLAS Muon Spectrometer}

In a difficult environment:

large magnetic field variations and temperature gradients
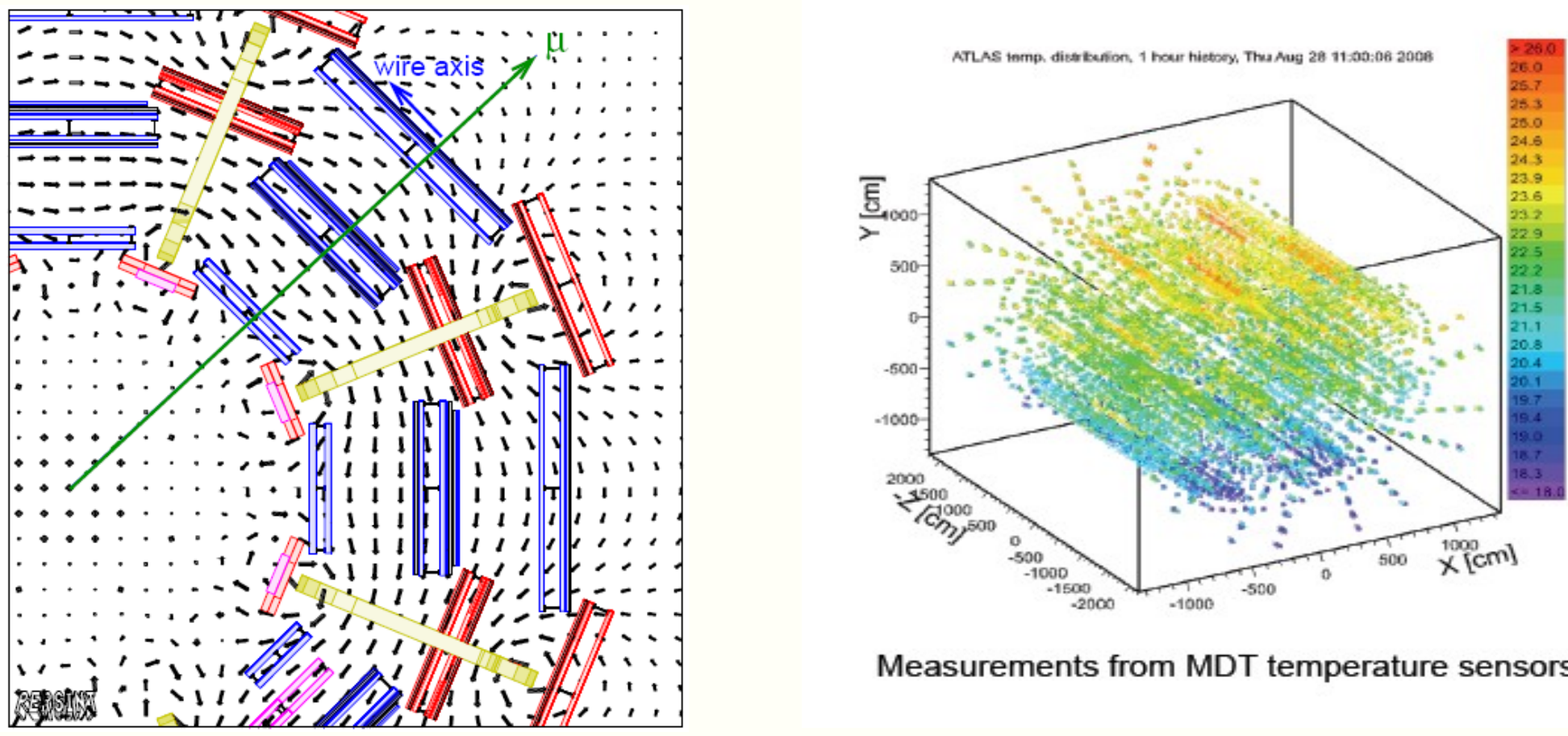

Measurements from MDT temperature sensors 


\section{The ATLAS Muon Spectrometer}

\section{Built to:}

- Provide an efficient muon trigger and Bunch Crossing (BC) identification

- Achieve high precision momentum measurement

\begin{tabular}{|c|c|c|c|c|c|c|c|c|}
\hline \multirow[b]{2}{*}{ Type } & \multirow[b]{2}{*}{ Function } & \multicolumn{3}{|c|}{ Champer resolution (RMS) in } & \multicolumn{2}{|c|}{ Measurements/track } & \multicolumn{2}{|c|}{ Number of } \\
\hline & & $z / R$ & $\phi$ & time & barrel & end-cap & chambers & channels \\
\hline MDT & tracking & $35 \mu \mathrm{m}(z)$ & - & - & 20 & 20 & $1088(1150)$ & $339 \mathrm{k}(354 \mathrm{k})$ \\
\hline $\mathrm{CSC}$ & tracking & $40 \mu \mathrm{m}(R)$ & $5 \mathrm{~mm}$ & $7 \mathrm{~ns}$ & - & 4 & 32 & $30.7 \mathrm{k}$ \\
\hline $\mathrm{RPC}$ & trigger & $10 \mathrm{~mm}(z)$ & $10 \mathrm{~mm}$ & ns & 6 & - & $544(606)$ & $359 \mathrm{k}(373 \mathrm{k})$ \\
\hline TGC & trigger & $2-6 \mathrm{~mm}(R)$ & $3-7 \mathrm{~mm}$ & $4 \mathrm{~ns}$ & - & 9 & 3588 & $318 \mathrm{k}$ \\
\hline
\end{tabular}




\section{Momentum measurement \& MDT resolution}

- the MDT single tube resolution is a function of the drift path;

- $\left\langle\Delta r>_{\text {single }}=80 \div 90 \mu \mathrm{m}\right.$, dependent on background, electronics and reconstruction algorithm;

- 3 [4] tubes $\rightarrow \Delta r_{\text {multilayer }} \approx 50 \mu \mathrm{m}$;

- needed for high $p_{\mathrm{T}}$ muons ;

- systematics must NOT spoil the overall accuracy;

$>$ calibrations and alignment better

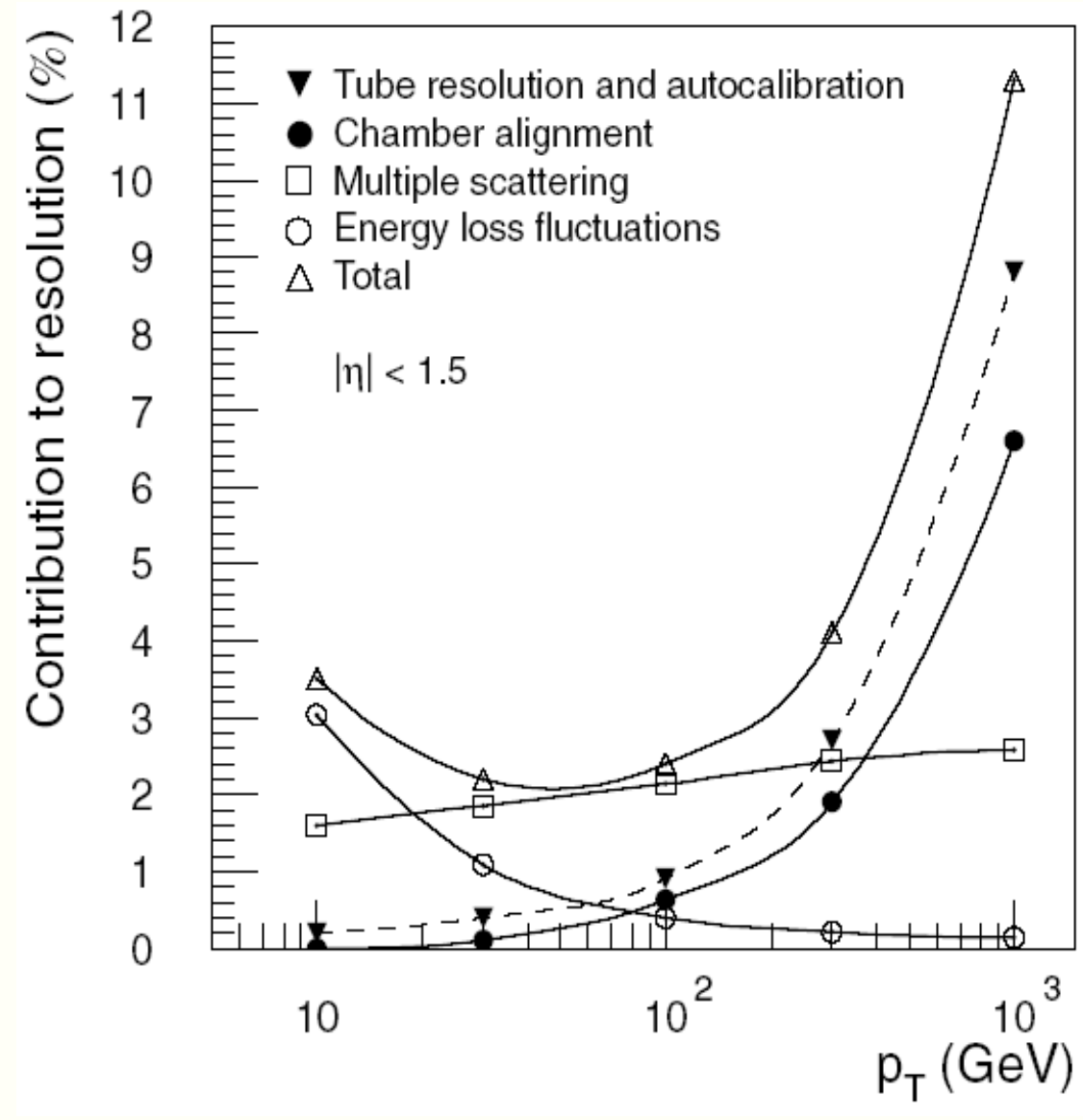
than $30 \mu \mathrm{m}$

Alignment system: 12232 optical sensors Autocalibration with dedicated data stream 


\section{Reconstruction}
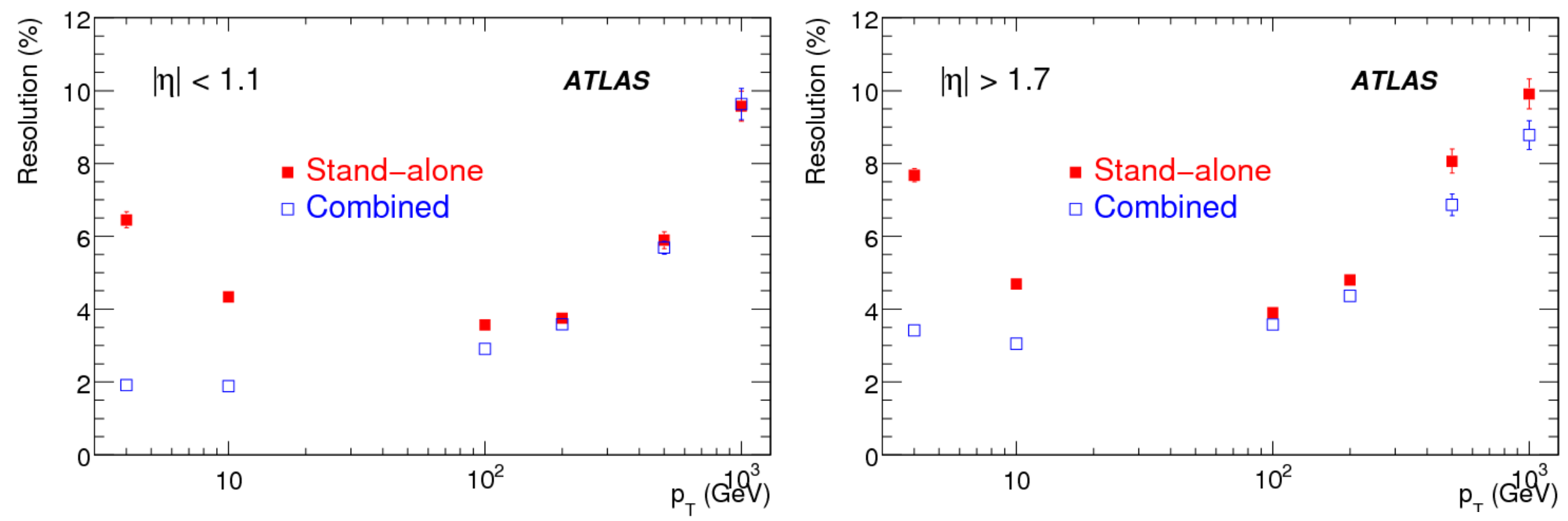

The Muon Spectrometer standalone reconstruction is complemented by the Inner Detector tracking

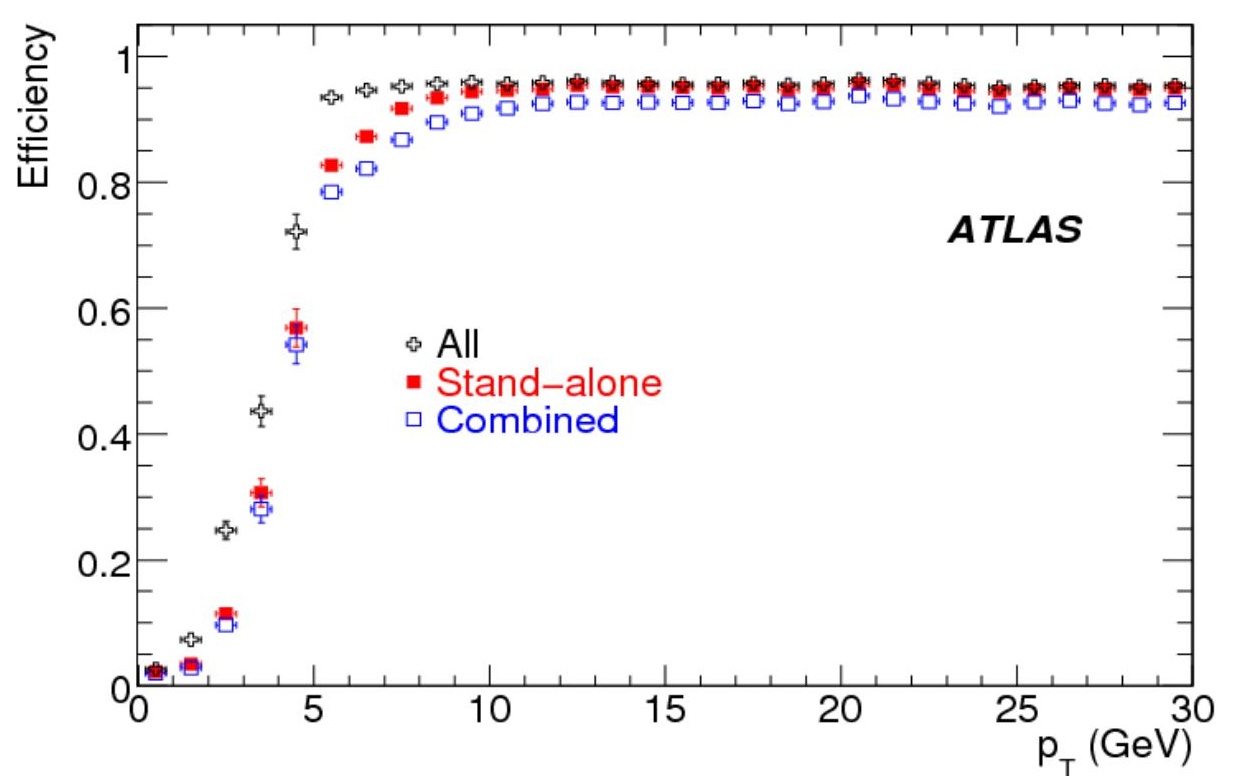




\section{Muon Spectrometer:}

\section{main source of cosmic triggers}

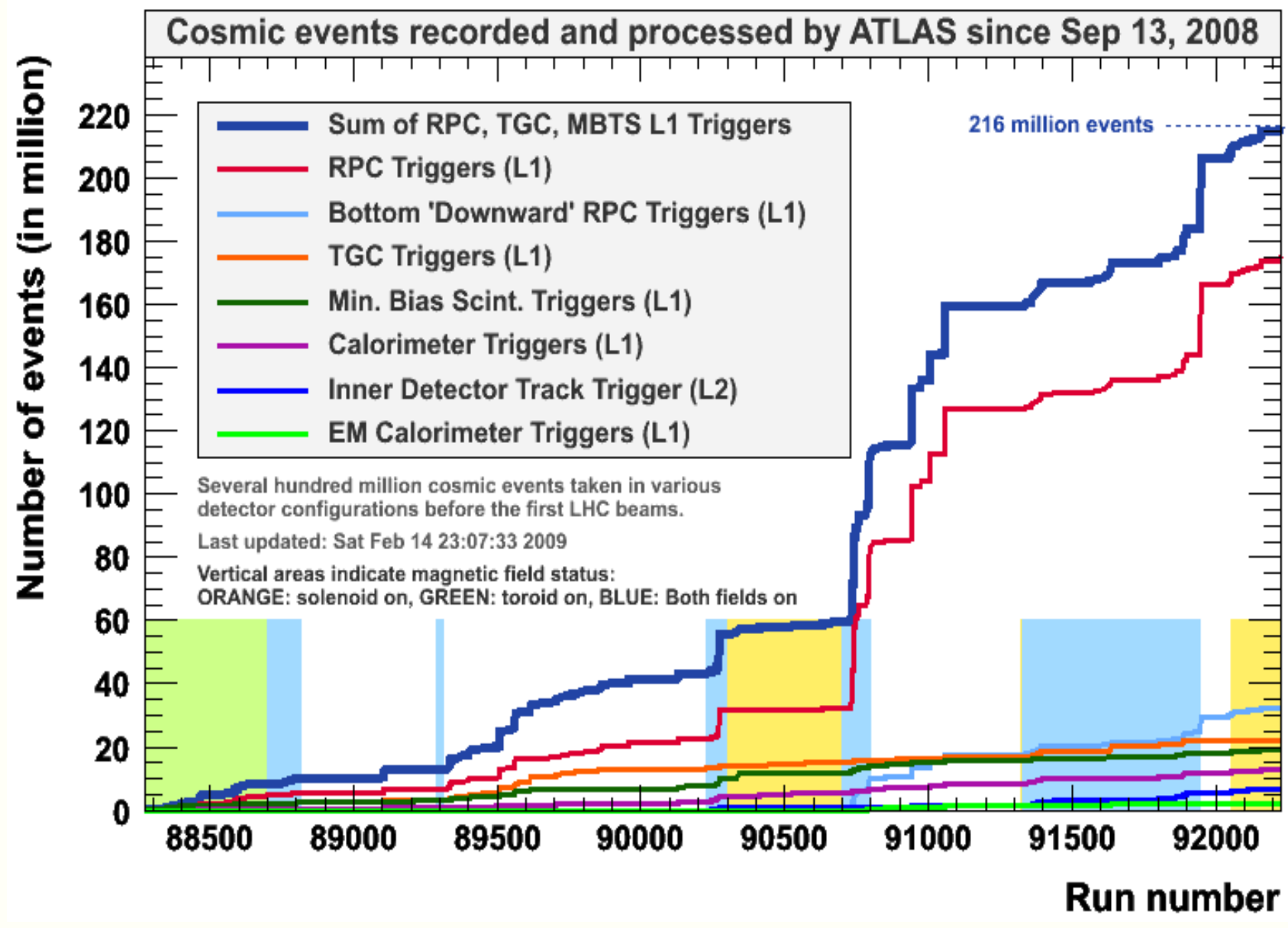

-High statistics collected in autumn 2008 (full ATLAS)

-Few high statistics runs studied in great detail -Subdetectors runs in 2009: RPC runs in January -"muon slice" runs at the end of April 


\section{Cosmic triggers}

- Sources of level 1 muon trigger:

- RPCs in the barrel,

- TGCs in the endcap

- Coarse time alignment

- In the barrel trigger based only on $\phi$ view, triggers from top sectors artificially delayed by $5 \mathrm{BC}$

- In the endcap both $\eta$ (bending) and $\phi$ (non-bending) view 


\section{$R P C$ system performance}

Dead strips $<2 \%$

Noisy channels $<1 \%$

In 2008 problems with

-Synchronzation

- Gas

- Temperature (reduced HV in top sectors)

Affected the overall efficiency ( $70 \%$ of trigger coverage)

Most of the problems were fixed during the winter shutdown, current trigger coverage is $95.5 \%$

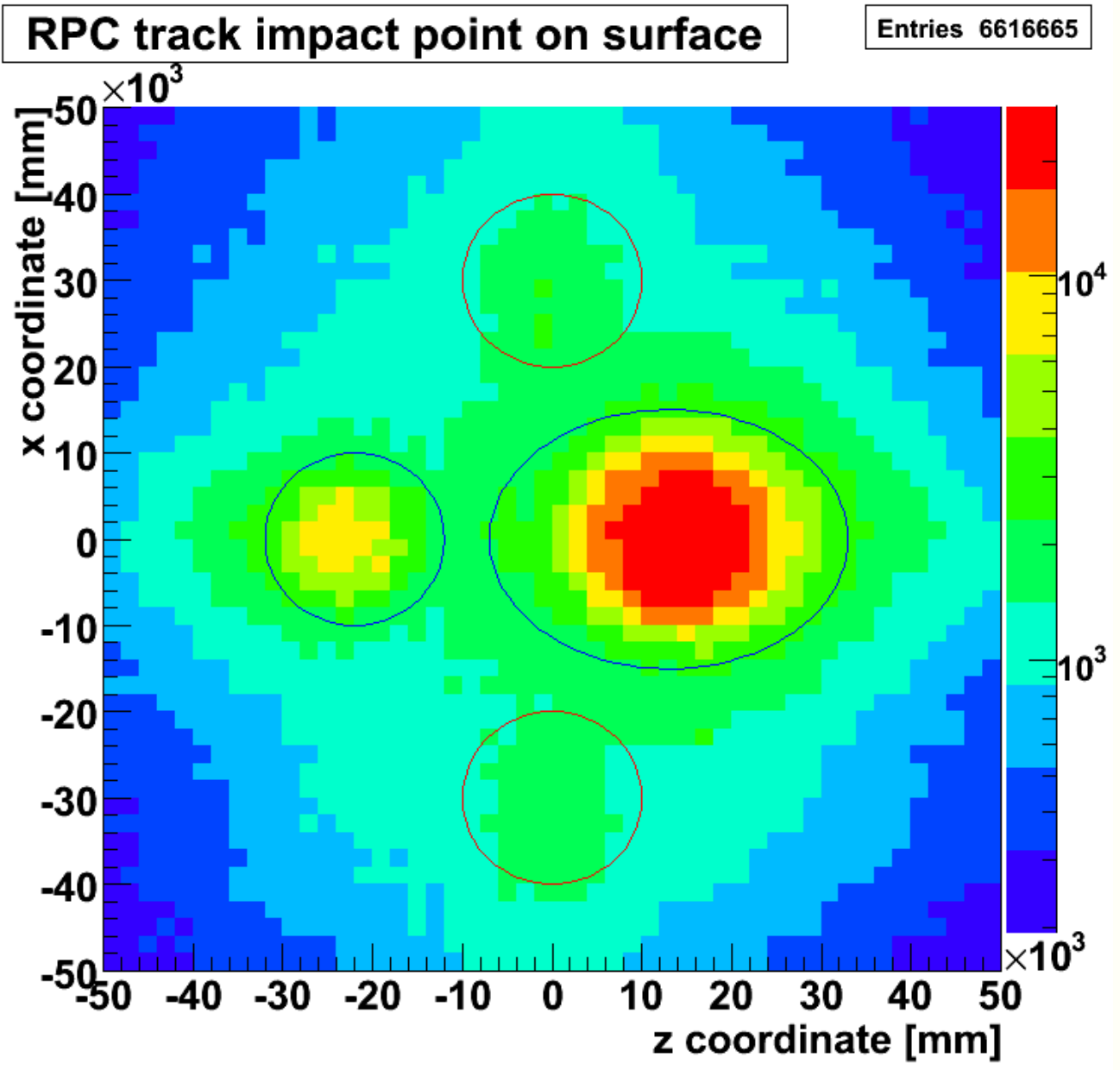




\section{$R P C$ trigger coverage}

\section{Low-pt trigger coverage}

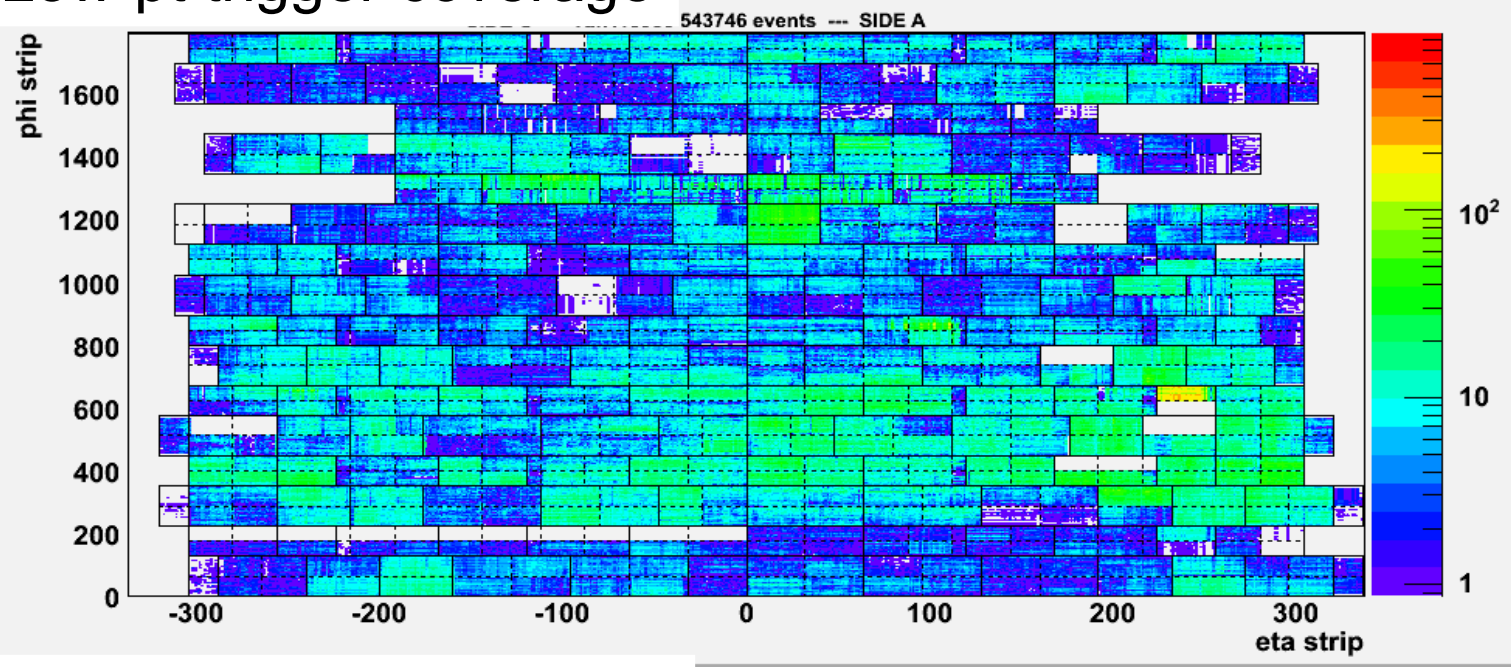

High-pt trigger coverage

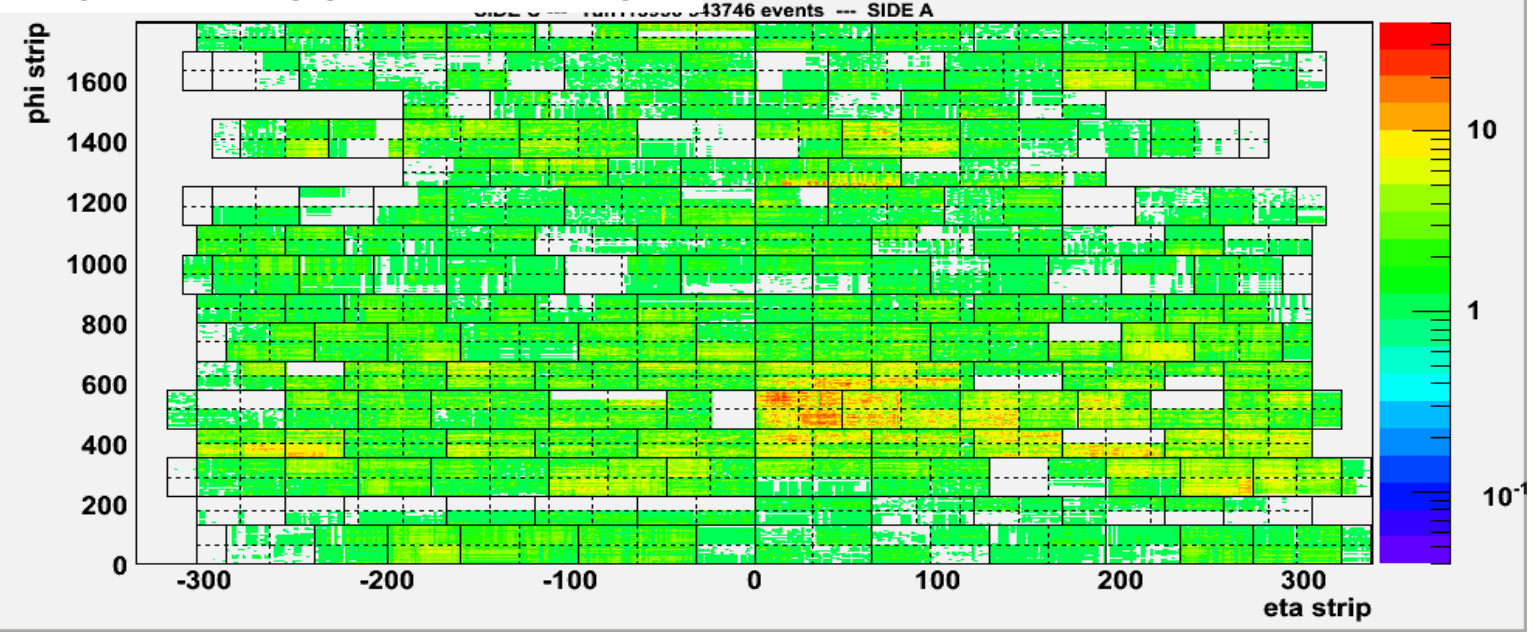

- Commisioning ongoing

- Trigger coverage increased from $70 \%$ in 2008 to current $95.5 \%$

- Full detector for 2009 run. 


\section{RPC trigger timing}

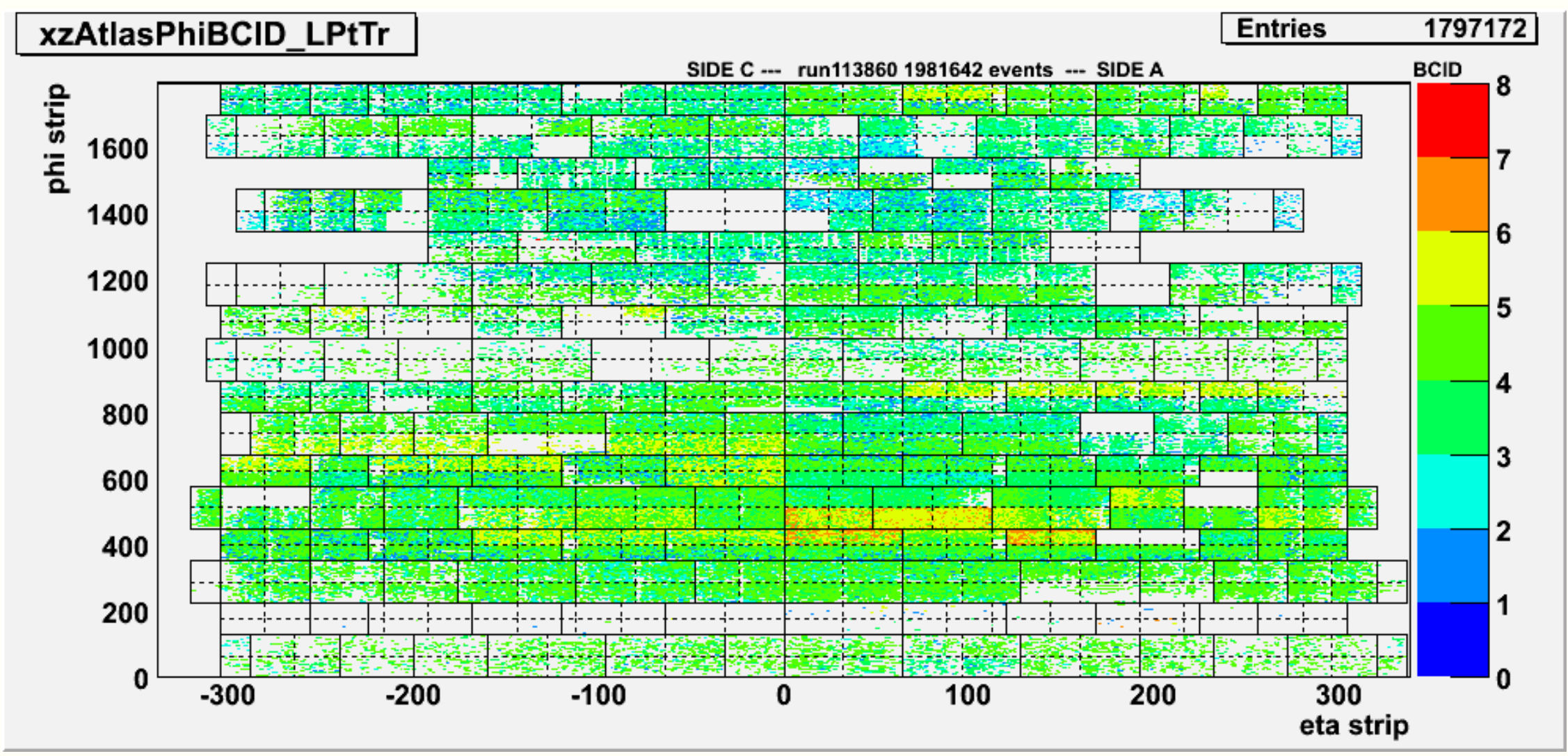




\section{$R P C$ performance}

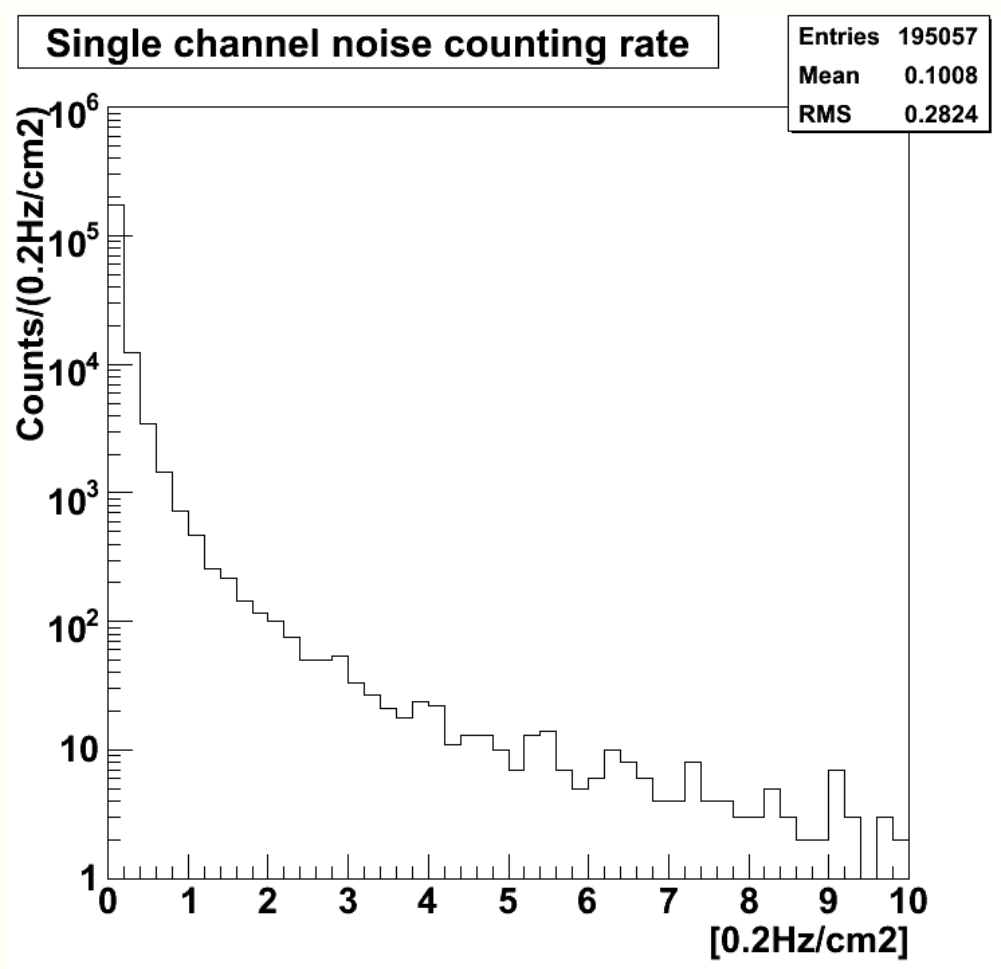

Random Trigger

Noise $=\mathbf{N}$ hits / (Ntrigger $\times$ RO-Window)/ strip Surf HV $=9600 \mathrm{~V}, \mathrm{Vth}=1000 \mathrm{mV}$ (Working Point)

Distribution of RPC hits per event with RPC cosmic trigger (not filled area) and with random trigger (black filled area).

$\mathrm{HV}=9600 \mathrm{~V}, \mathrm{Vth}=1000 \mathrm{mV}$ (Working Point)

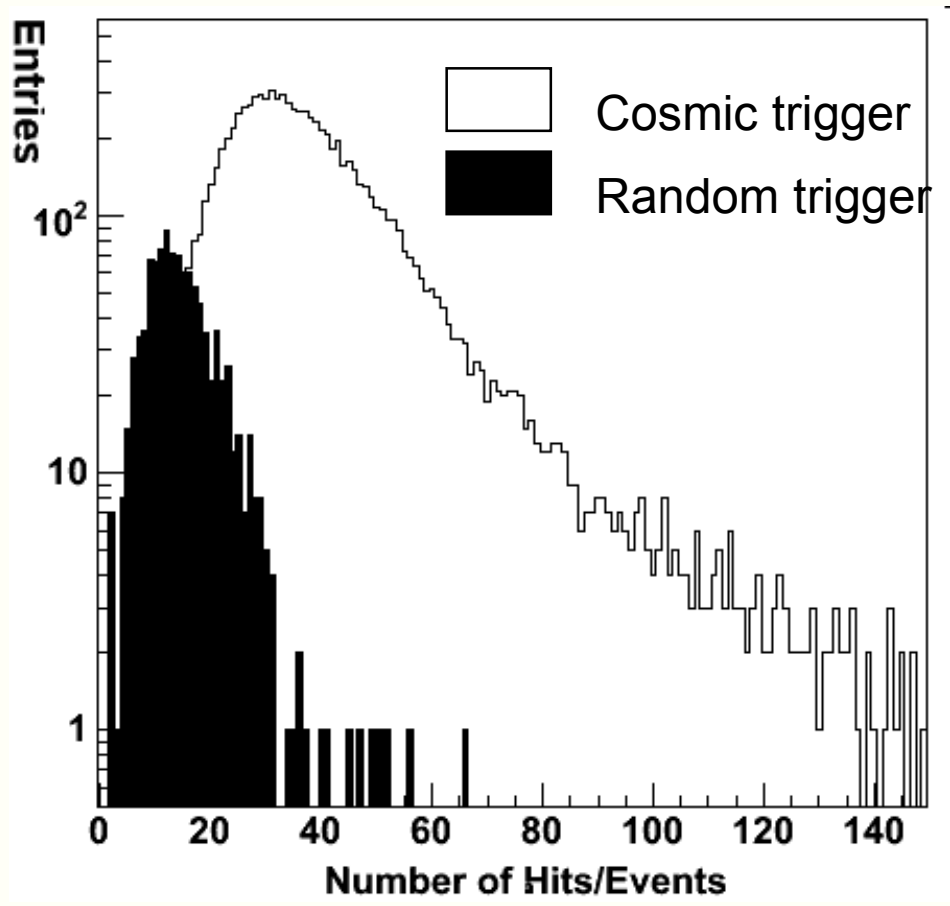




\section{$R P C$ performance}

Efficiency vs HV
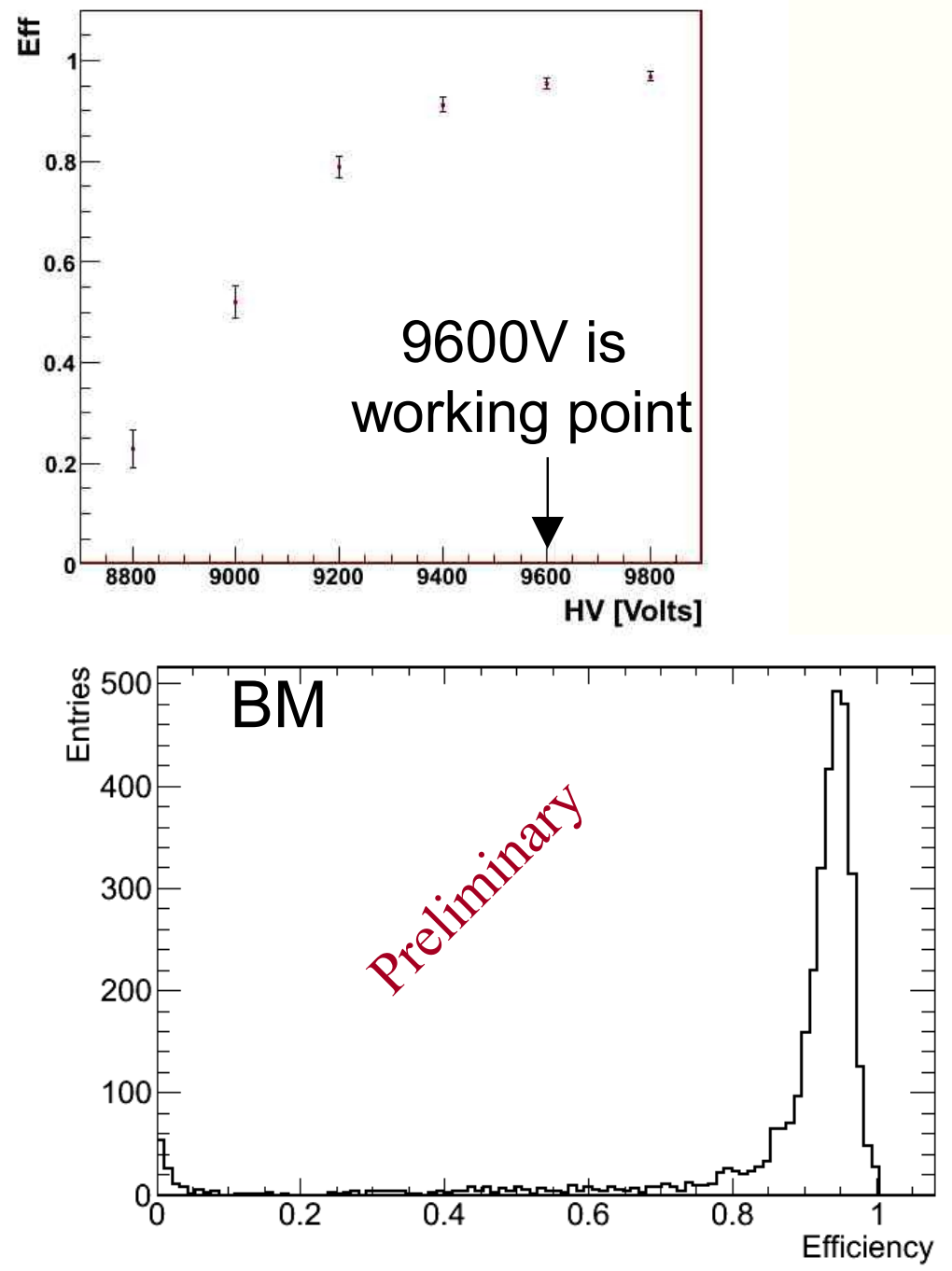

Cluster size vs HV
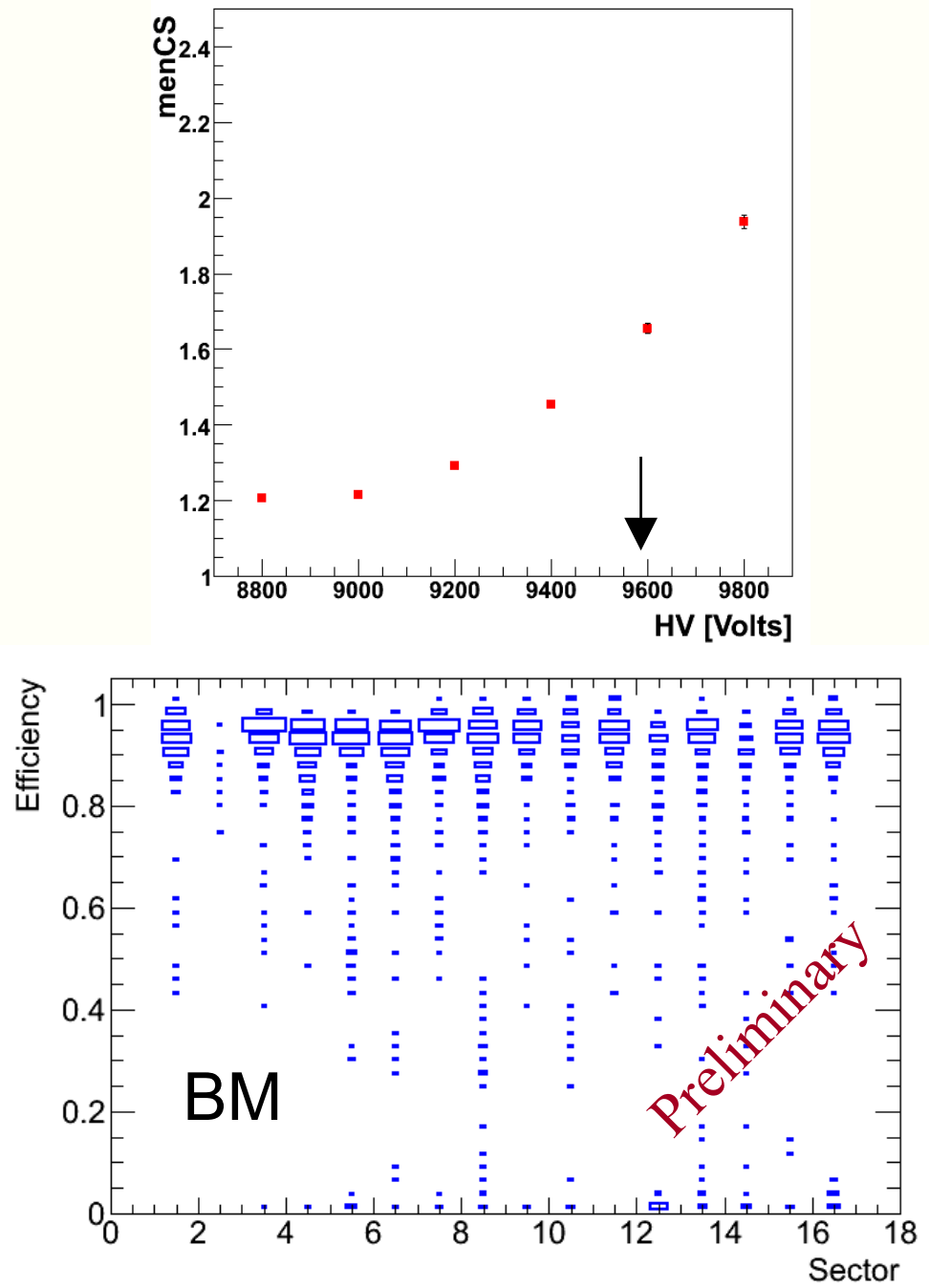


\section{TGC system performance}

- $99.8 \%$ of chambers operational

- Single dead channels $<0.01 \%$

- Clustered dead channels $<0.1 \%$

- Noisy channels ( $>2.5 \%$ occupancy) $<0.04 \%$

\section{XY_View_C}

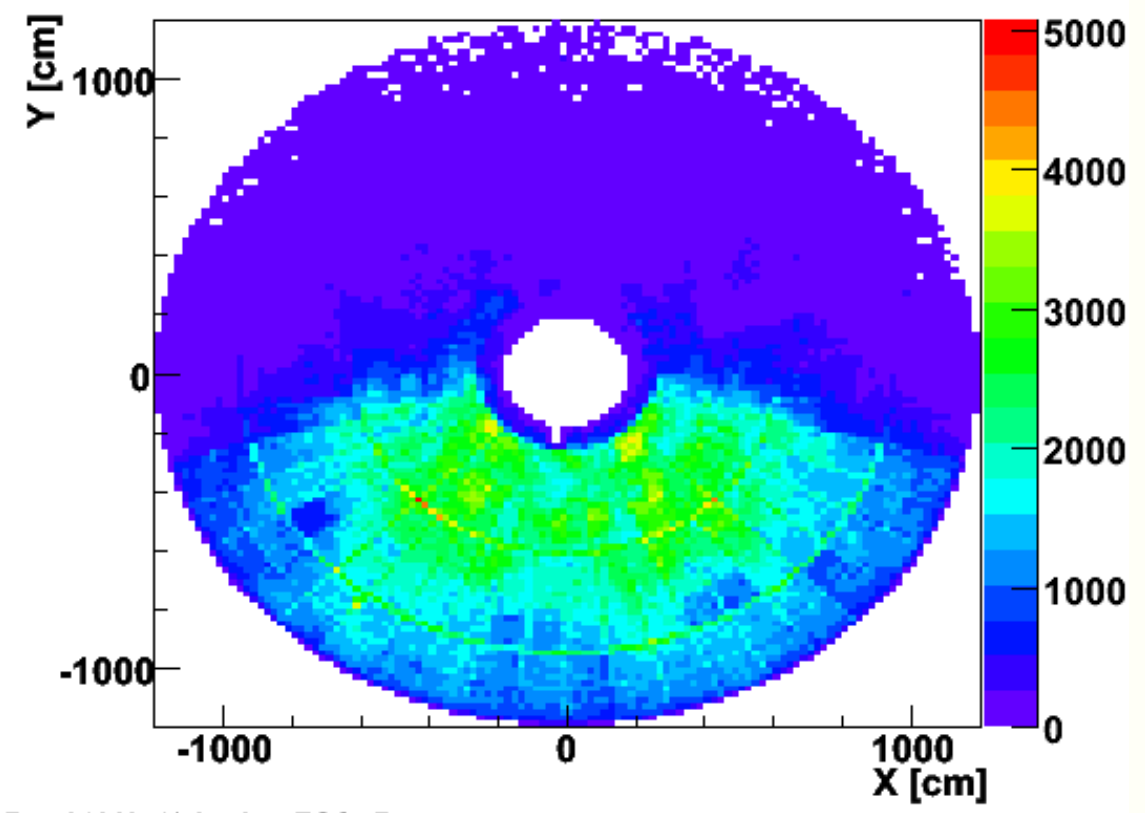

\section{XY_View_A}

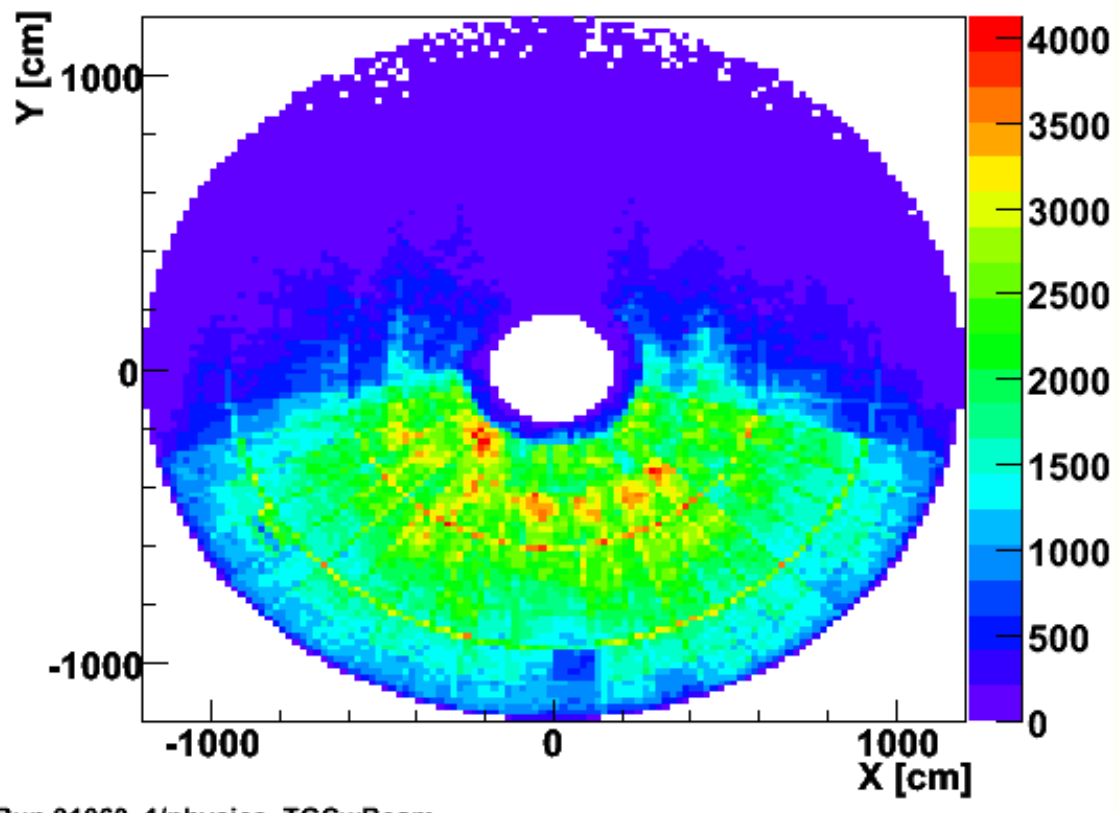

Run 91060,1/physics_TGCwBeam

/MuonDetectors/TGC/XY_View_A

- Full trigger coverage

- All sectors read-out, bottom $1 / 3$ used for triggering

Run $91060,1 /$ physics TGCwBeam /MuonDetectors $/ \mathrm{TGC} / \bar{X} Y$ _View_C 


\section{TGC performance}

TGC detector efficiency
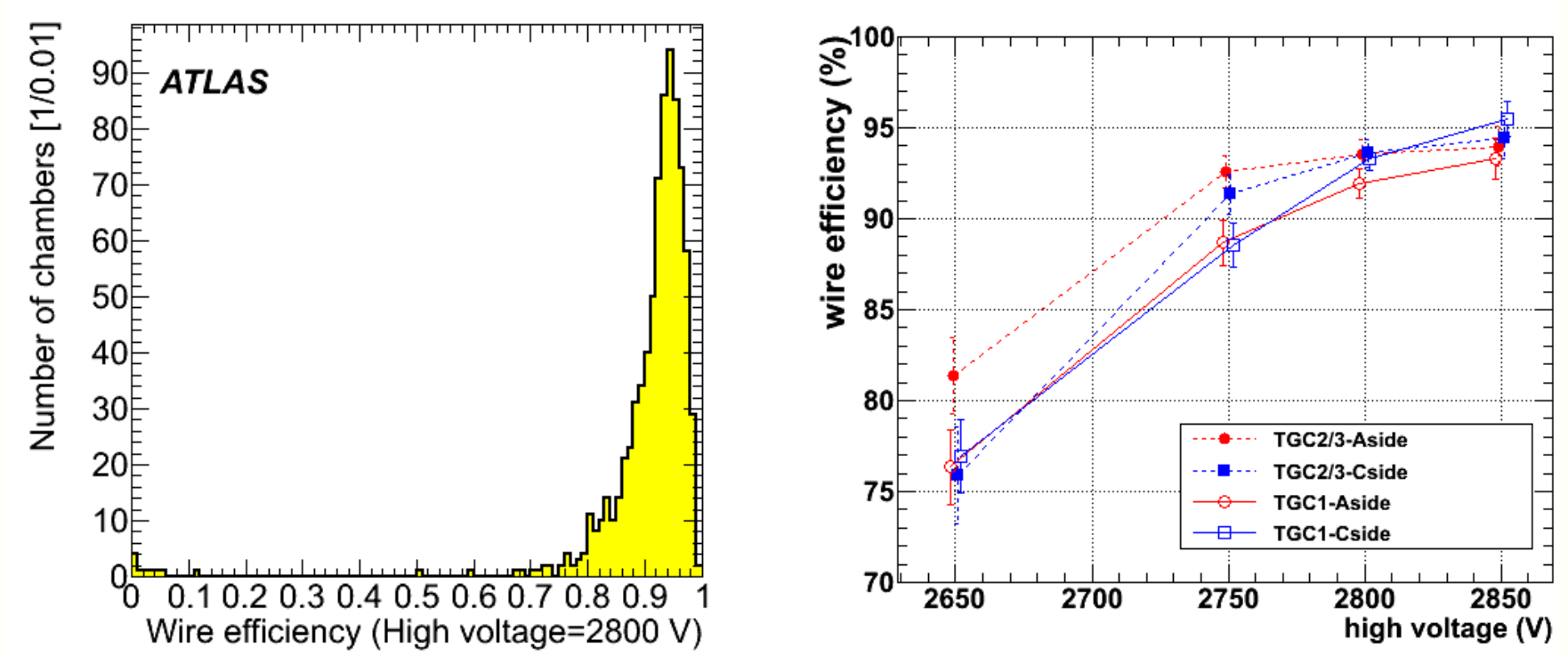

TGC trigger synchronization
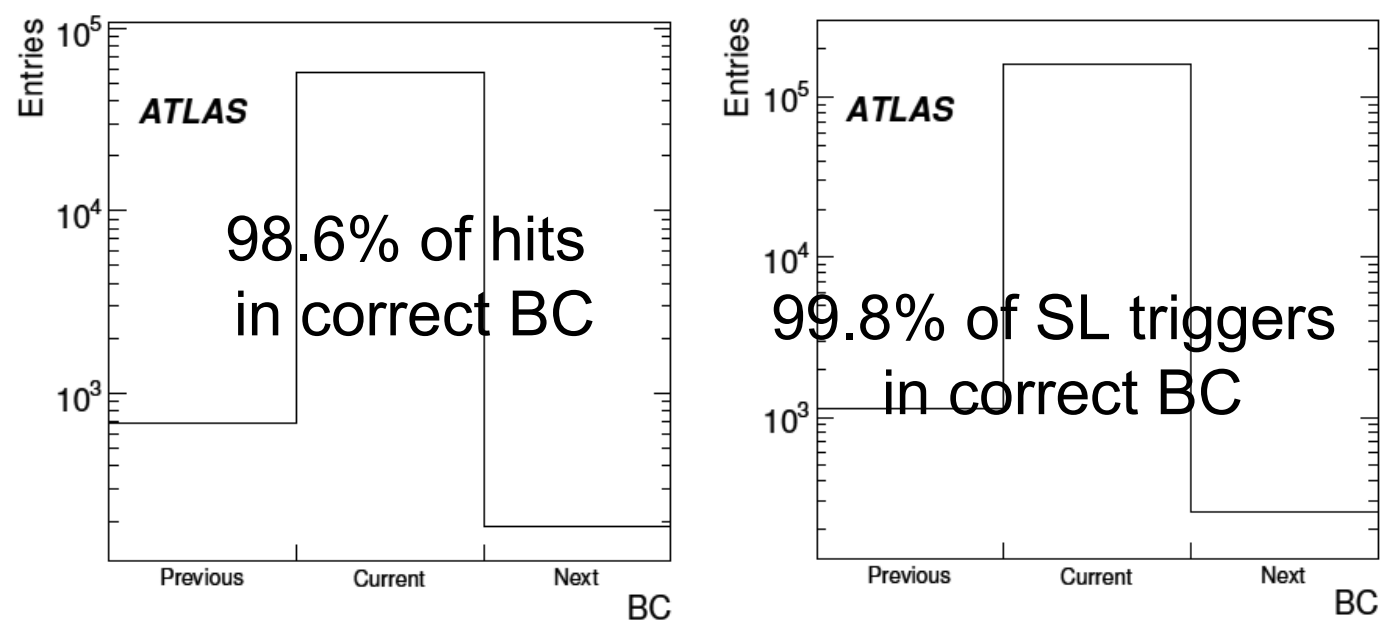


\section{MDT system performance}

Statistics/chamber in one long 2008 run

Out of 1100 chambers:

- 2 chambers not yet installed

- 3 chambers not readout (gas system problems)

- 2 chambers with HV off

- 32 chambers with HV off for 1 of the 2 multilayers

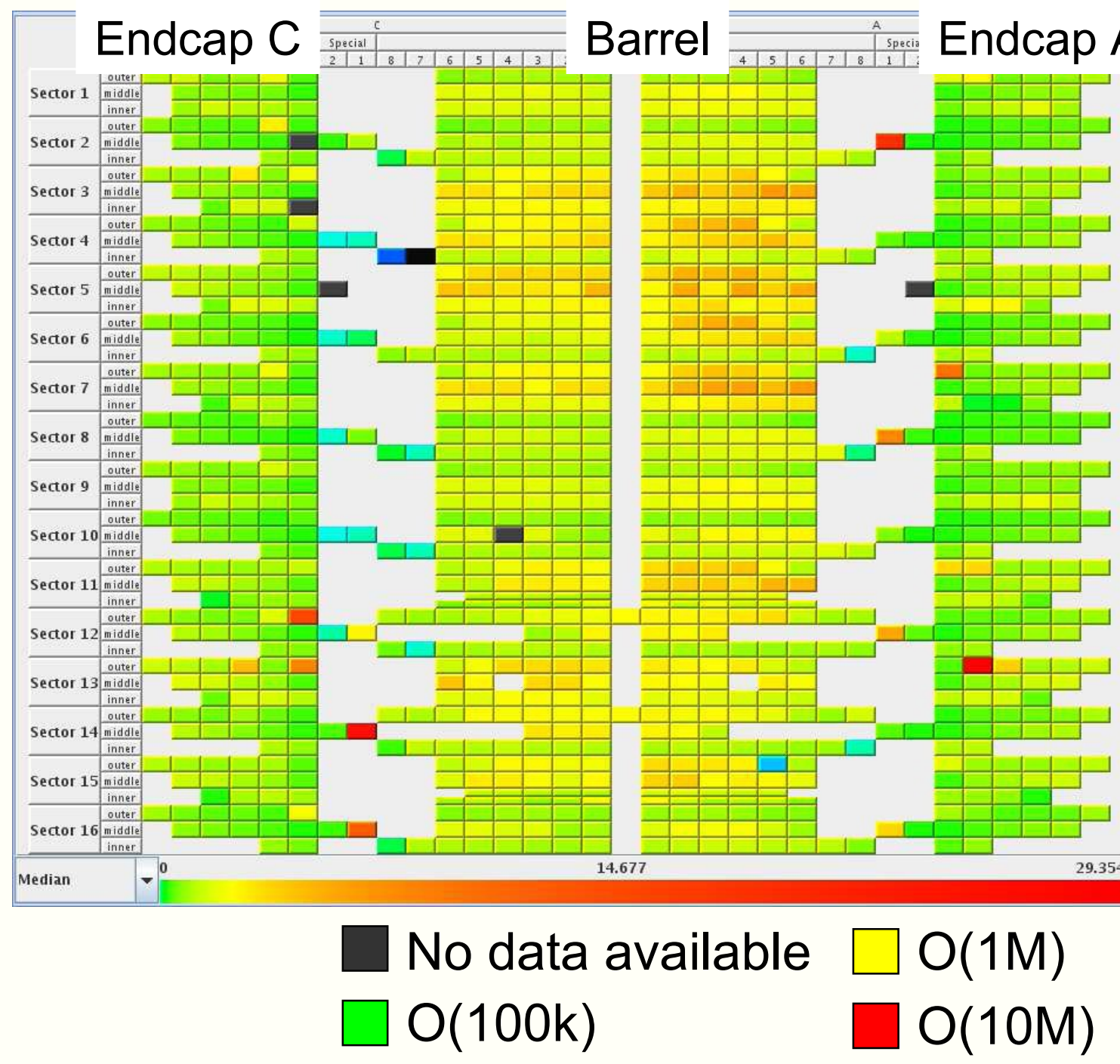




\section{MDT efficiency}
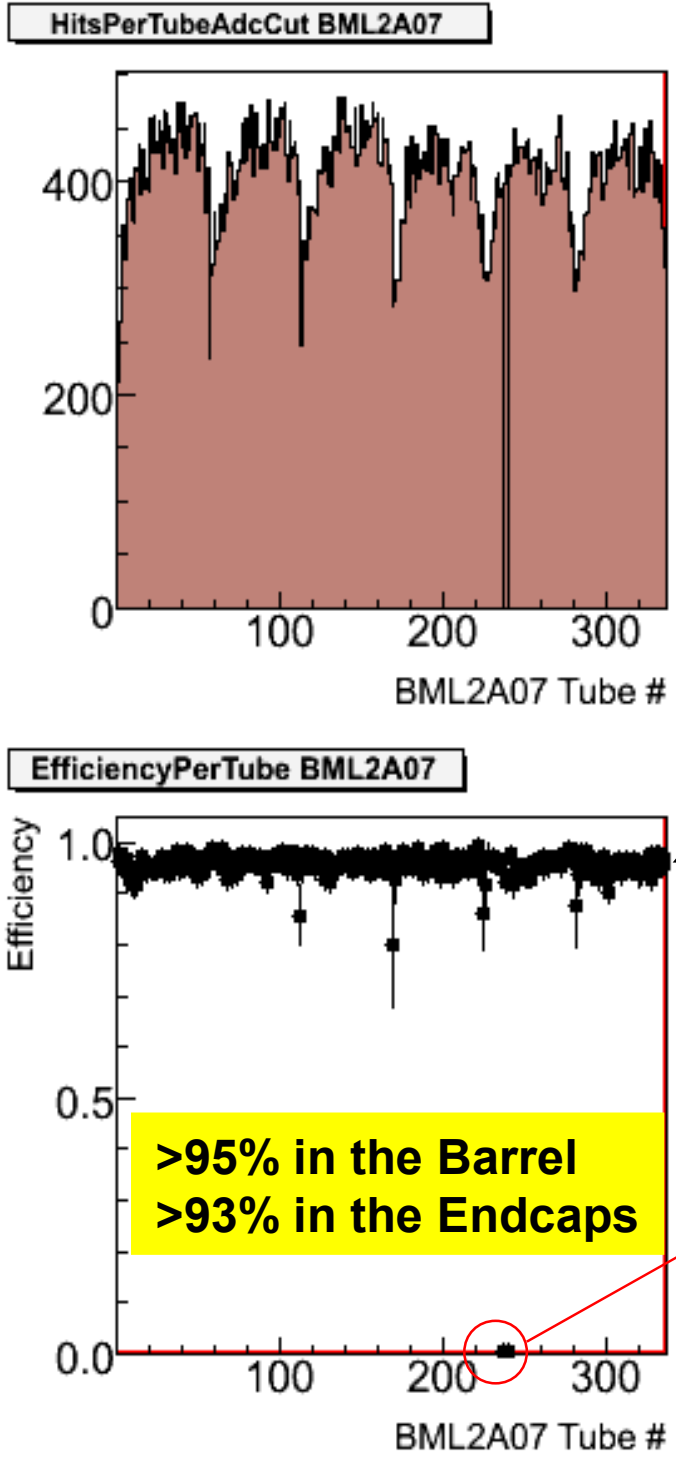

MDT BI Segment efficiency

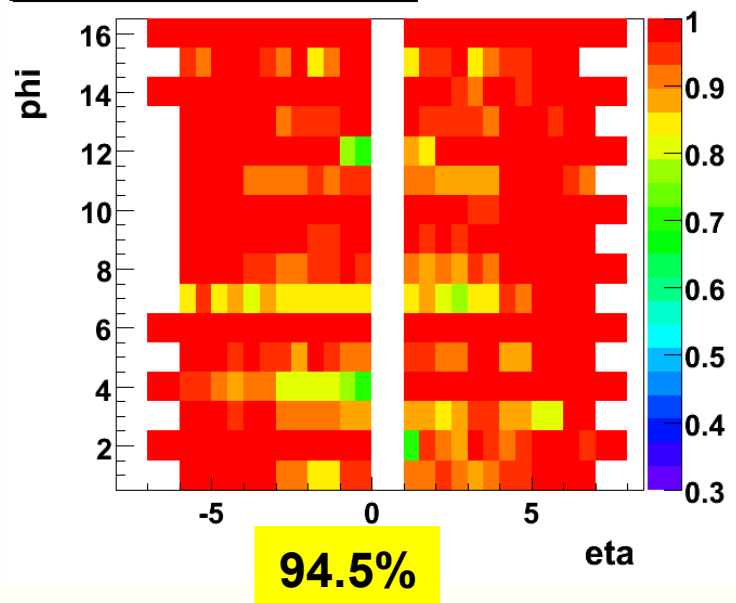

$94.5 \%$
MDT BM Segment efficiency

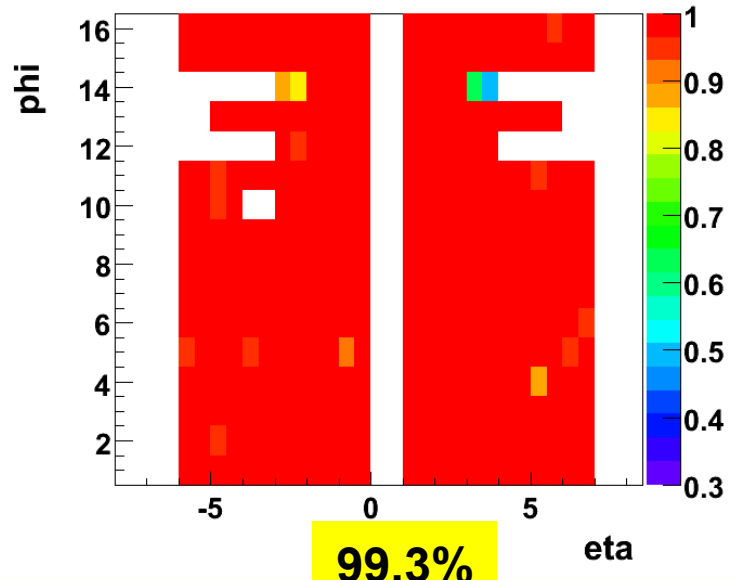

Tube \& segment efficiencies in cosmic ray events

$\cdot 0.1 \%$ single dead channels

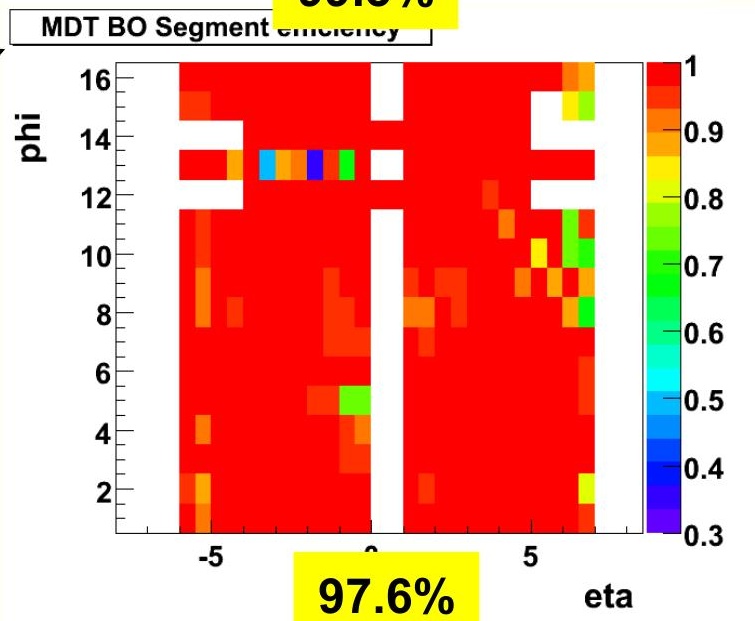

- $1 \%$ clustered dead channels (being fixed)

-Noisy channels ( $>5 \%$ occupancy) $<0.2 \%$ 


\section{Alignment}

In autumn 2008 :

-the barrel optical system was fully installed

$\bullet 99.7 \%$ of the sensors were functioning correctly

\begin{tabular}{lrrr}
\hline Type & Total & Working & Broken \\
\hline Projective & 117 & 117 & 0 \\
Axial & 1036 & 1031 & 5 \\
Praxial & 2010 & 2008 & 2 \\
Reference & 256 & 253 & 3 \\
CCC & 260 & 260 & 0 \\
BIR-BIM & 32 & 32 & 0 \\
Inplane & 2110 & 2101 & 9 \\
\hline Total & 5817 & 5798 & 19 \\
$\%$ & & 99.7 & 0.3 \\
\hline
\end{tabular}

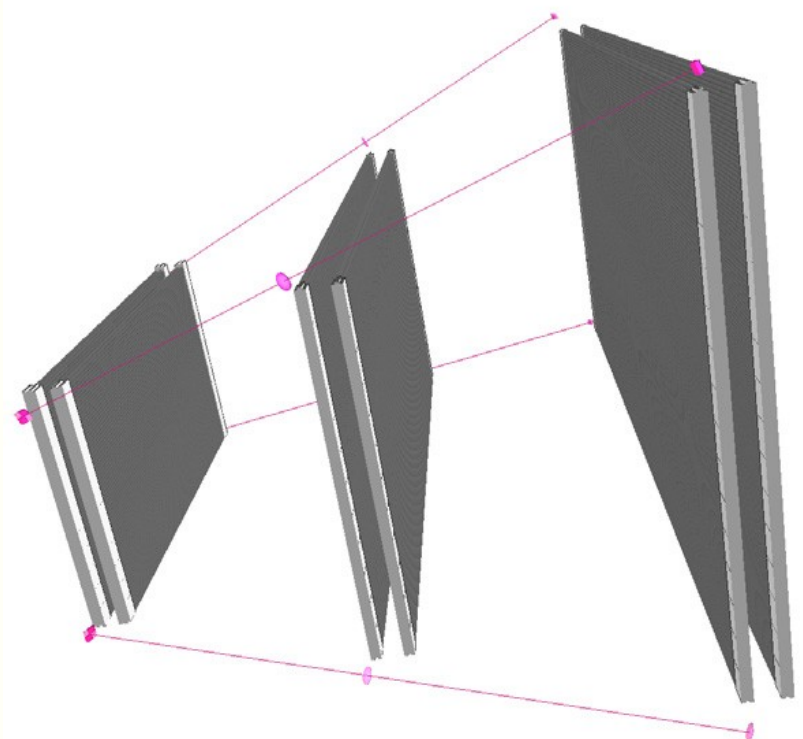

-99\% of all relevant alignment sensors in the endcaps were functioning

-only very few failed during the several months of data-taking in 2008.

-The effect of the missing sensors on the final alignment quality was negligible.
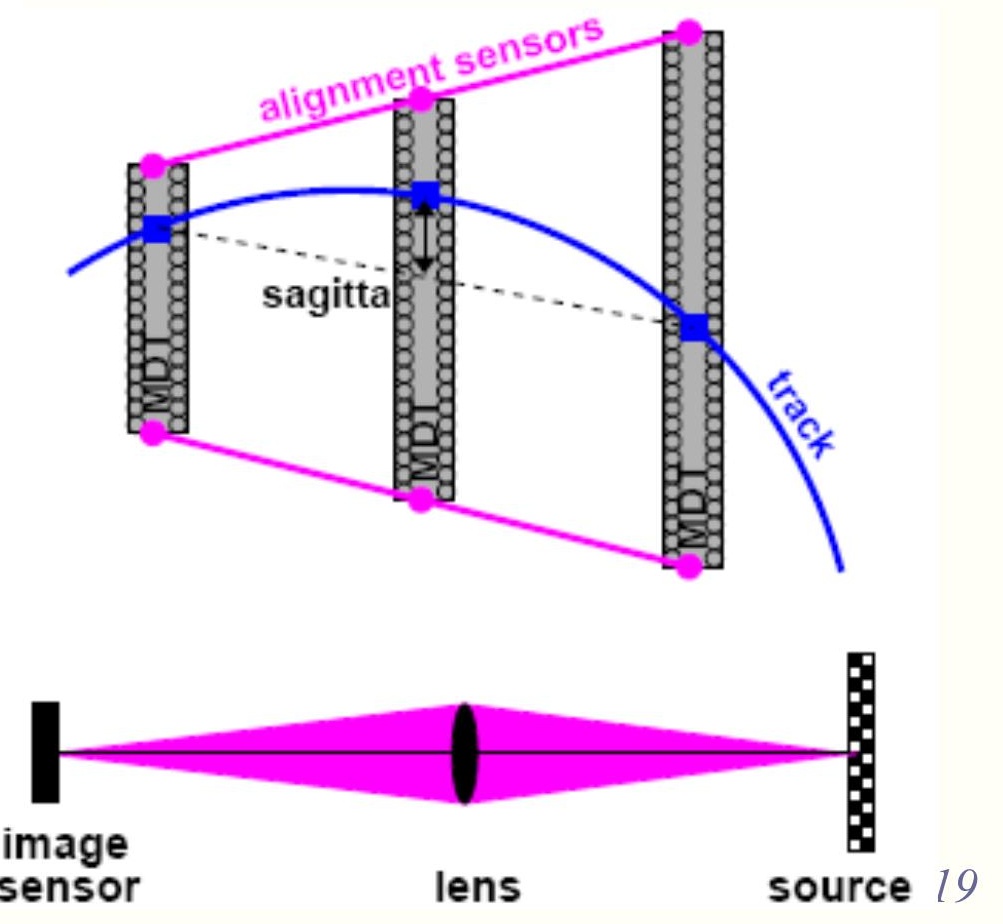


\section{Alignment system performance}

\section{BARREL}
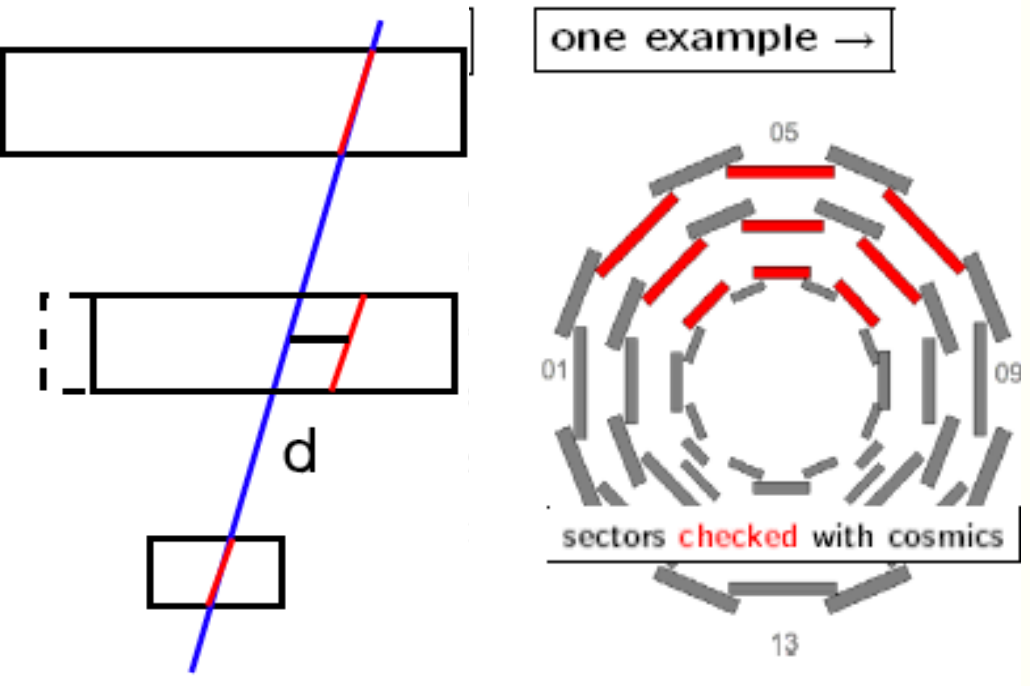

-Barrel Optical Alignment: at $200 \mu \mathrm{m}$ level for large sectors $(0.5-1 \mathrm{~mm}$ for small ones)

-Track based alignment : improvement to $<50 \mu \mathrm{m}$ level
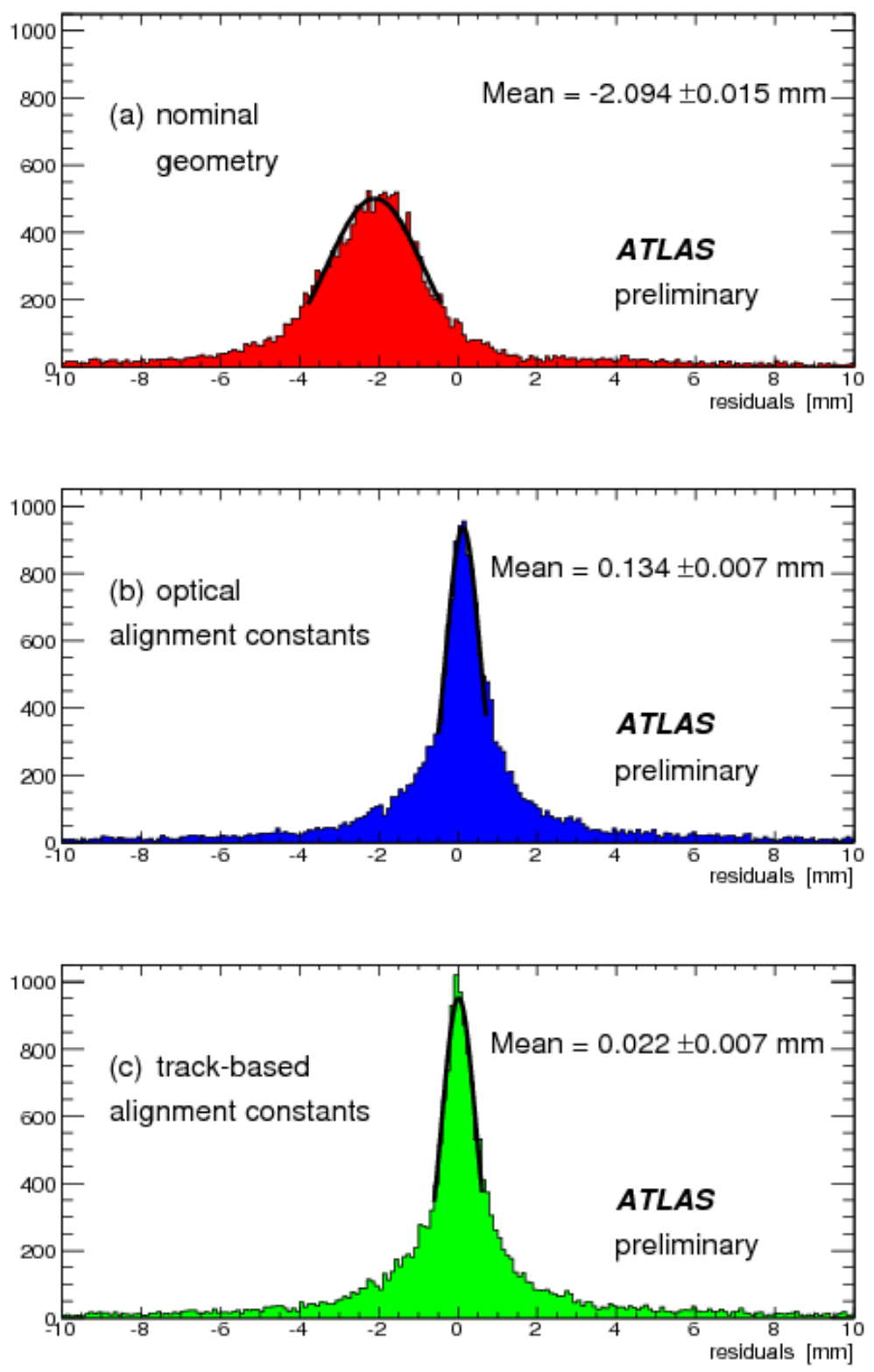


\section{ENDCAP}

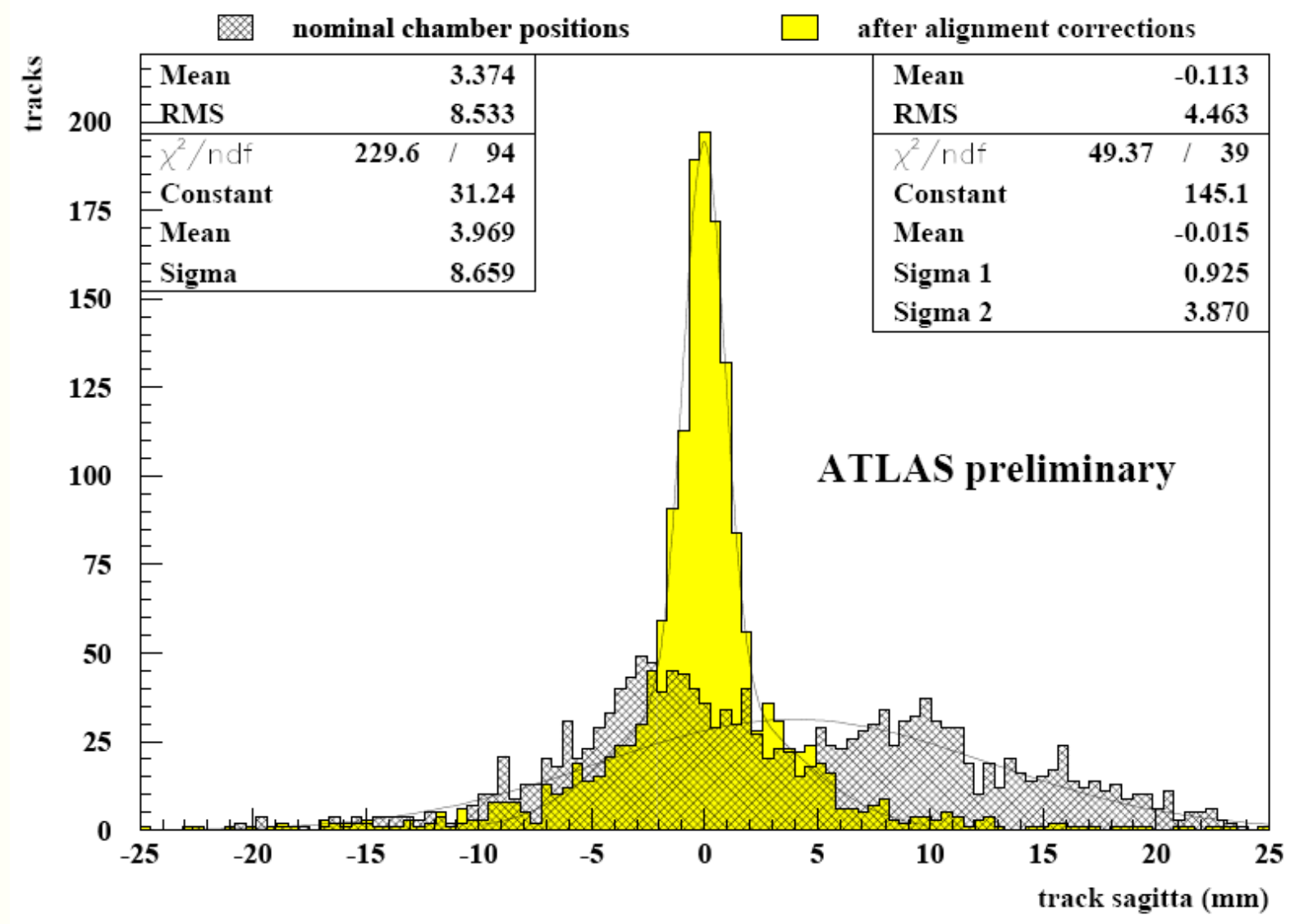

\section{Endcap Optical Alignment:}

sagitta residuals within the design specification

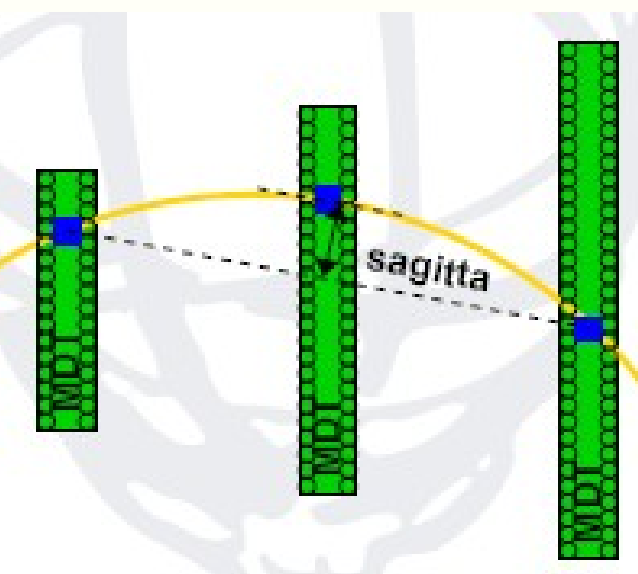




\section{MDT calibration}

- MDT tubes are calibrated using the muon tracks through the iterative auto-calibration procedure

- A large number of tracks is needed and a dedicated high rate stream extracted from Level 2 trigger has been foreseen for this purpose for $\mathrm{p}-\mathrm{p}$ collisions

- The stream has been regularly extracted and distributed to the 3 Calibration Centers (Ann Arbor, Munich, Rome) during the cosmic data taking to test the full calibration chain

- But calibrations for cosmic runs were produced from the complete ATLAS events to benefit from the larger statistics of non pointing tracks

- The space-time relation in the chambers with too low statistics has been obtained from a dedicated chamber located at the surface and connected to the same gas distribution ("gas monitoring chamber") 


\section{MDT to}

- The arrival time of the cosmics is asynchronous with respect to the $40 \mathrm{MHz}$ clock of the experiment which gives the time reference for the time digitization

- The $t 0$ for the MDT should therefore be computed on an event by event basis

- Either from the time measured by the triggering detector

- Or extracting it from a fit to the drift times of the hits on a segment

- The second approach was adopted in the cosmic analysis to recover also the effect of the not yet optimal time alignment of the trigger detectors

- To help the fit preliminary rough t0 values were obtained for each chamber from the drift time spectra 


\section{CSC system performance}

- $98.5 \%$ of chambers operational

- Dead channels $<0.1 \%$

- Calibration data collected

- ROD firmware problem prohibited full integration in ATLAS DAQ during 2008

- Fix on the way for 2009 run 


\section{Muon Spectrometer performance}

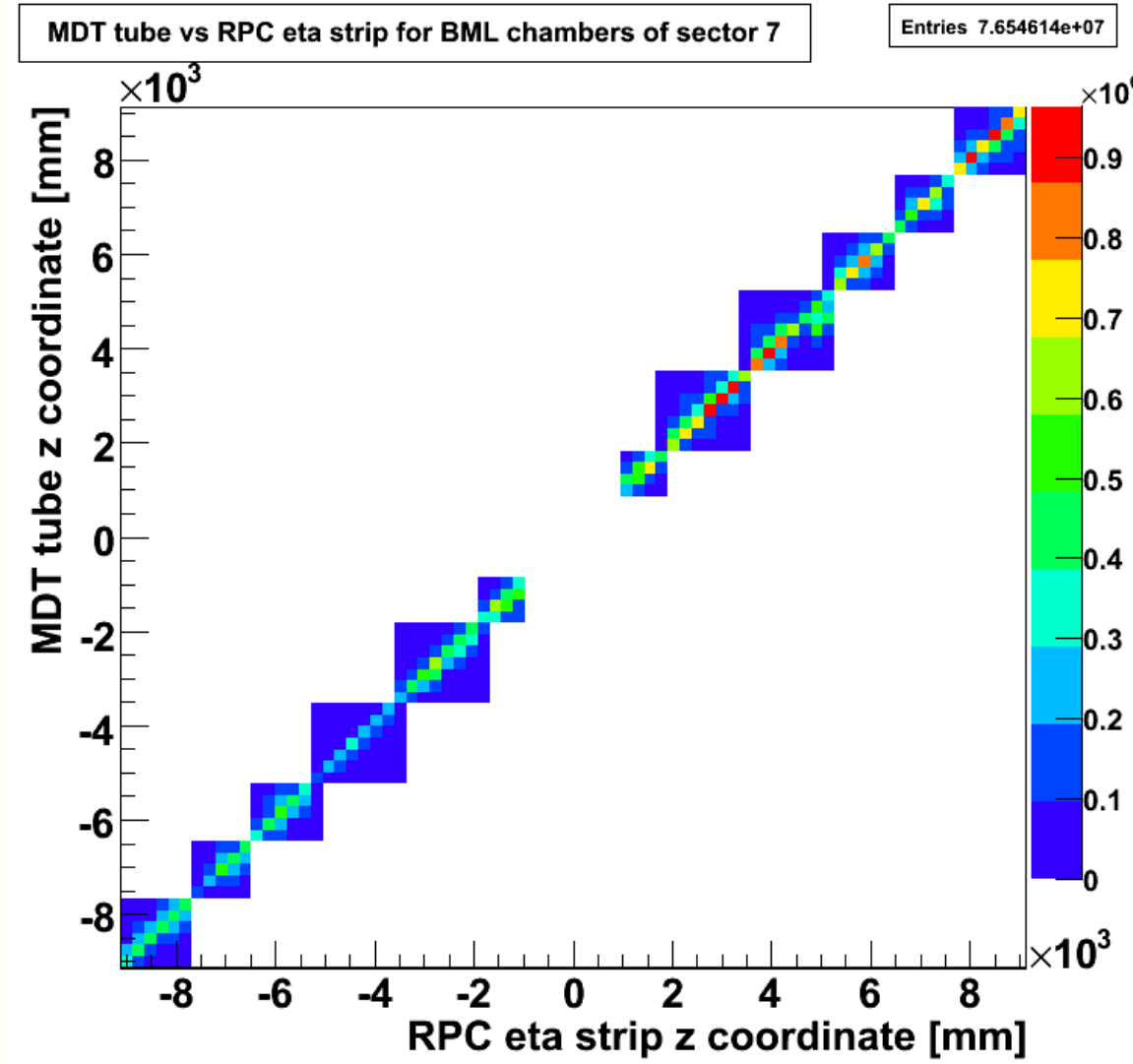

Correlation between MDT hits and RPC eta strips

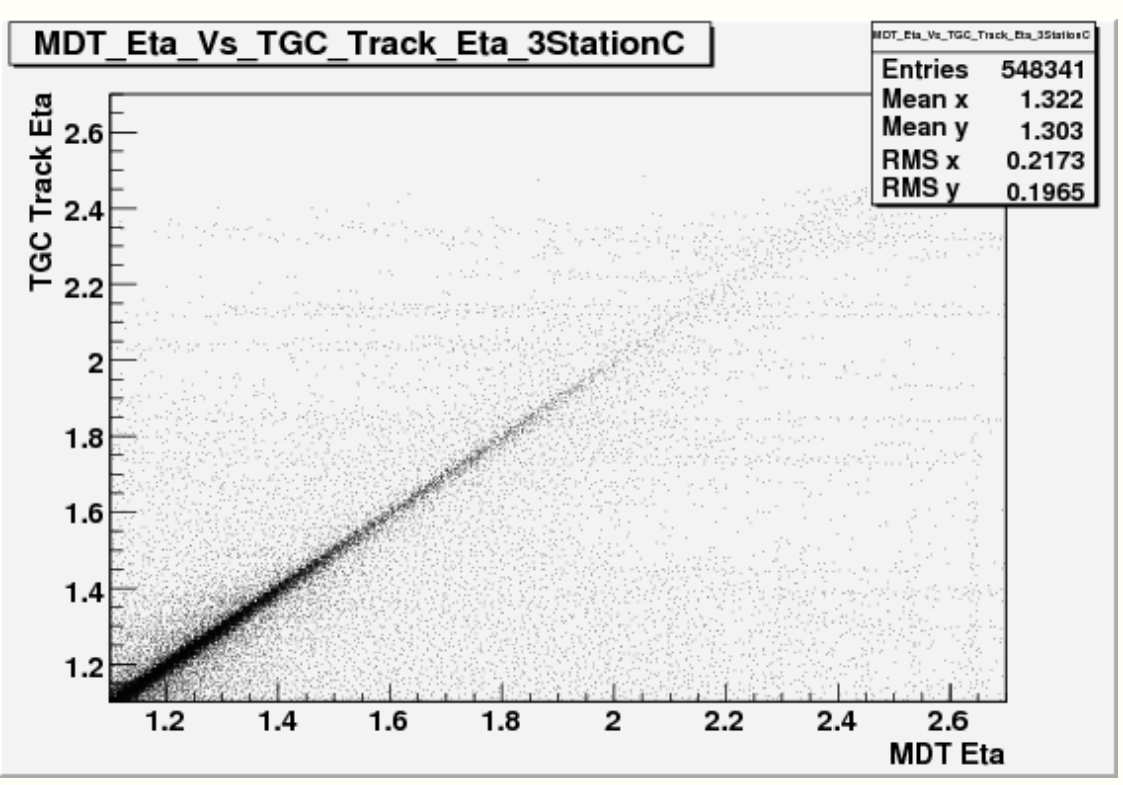

Reconstructed TGC tracks interpolated to MDT middle layer vs MDT hit 


\section{Tracking}

Momentum comparison between "pointing" standalone tracks reconstructed in the top "hemisphere" and in the bottom one ( $\mathrm{P}_{-}$top $<25 \mathrm{GeV}$ )

$6 \mathrm{GeV} / \mathrm{c}$, as expected for tracks crossing twice the calorimeters, and in agreement with $3 \mathrm{GeV} / \mathrm{c}$ between Muon standalone and Inner Detector momenta

A "Tag\&Probe" method to measure efficiencies in one hemisphere when a good track (eventually combined to the Inner Detector one) is present in the opposite one is currently under development.
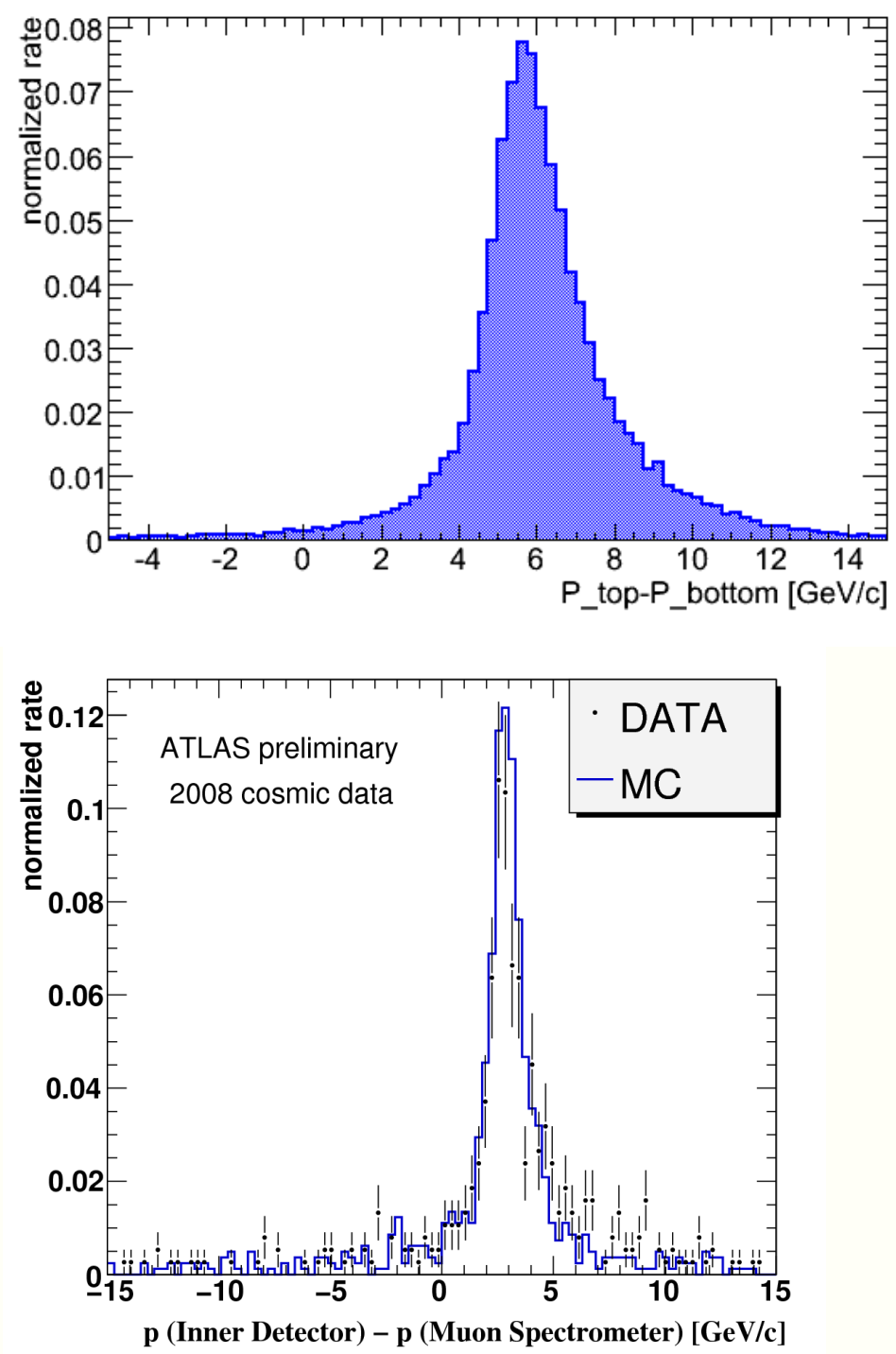


\section{MUON - Inner Detector}

\section{Comparison}

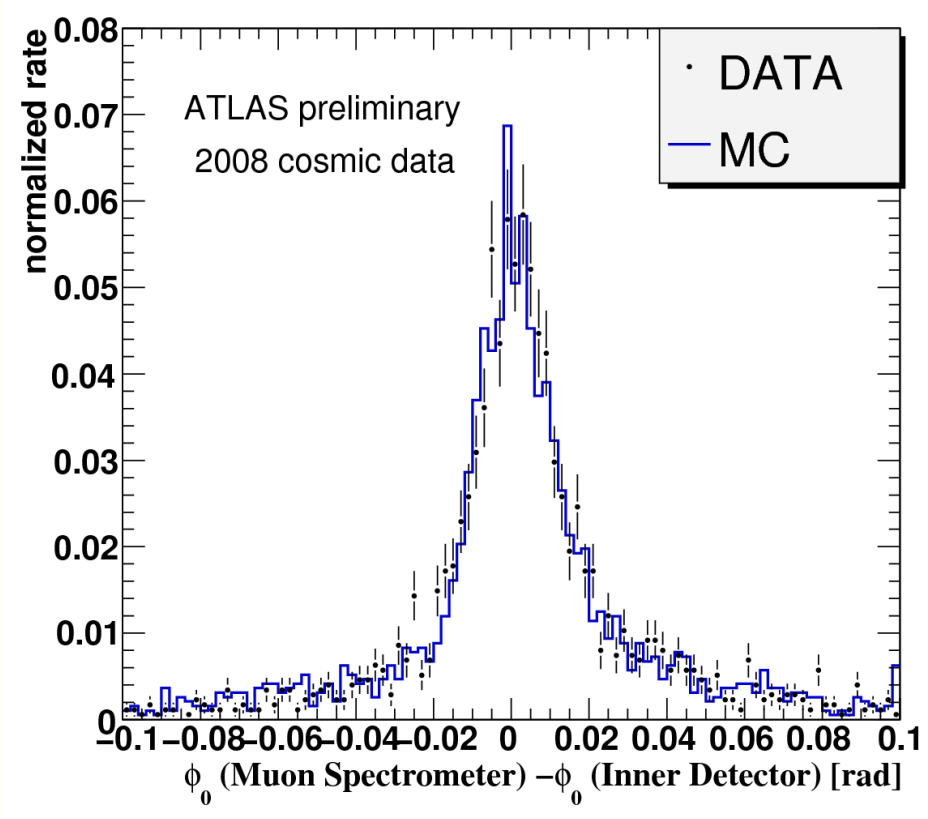

NB : very preliminary alignment and no relative MUON-

\section{Inner Detector} alignment yet!

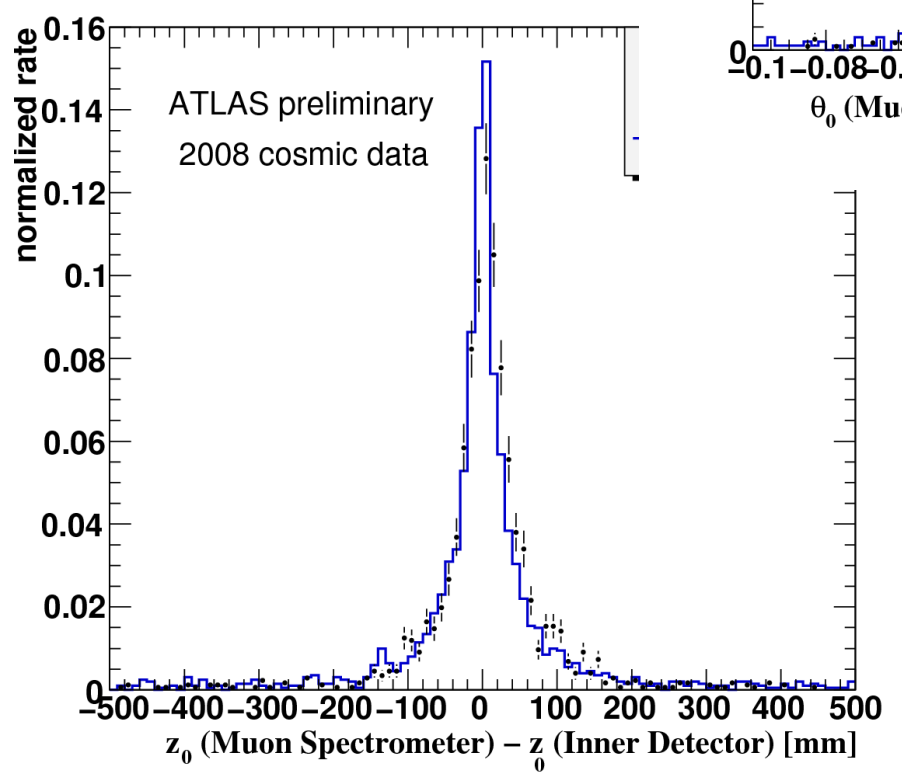

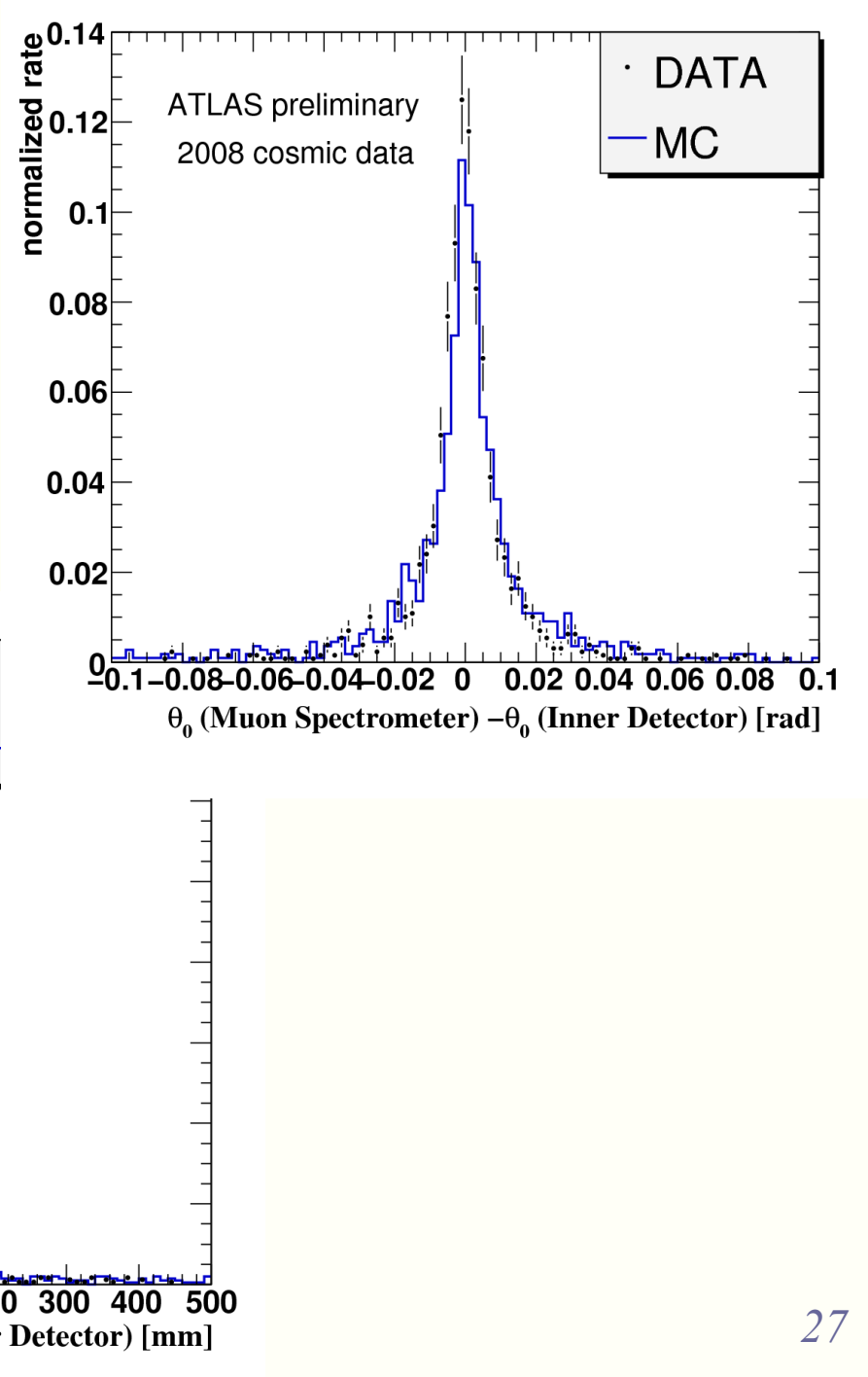




\section{Conclusion}

- The cosmic events collected in 2008, mostly with muon triggers, are used for detailed studies of the Muon Spectrometer detector and standalone reconstruction.

- Most of the problems observed in the 2008 cosmic runs have been understood and fixed.

- Cosmic data to be collected next summer, with a full trigger coverage and an improved time alignment, will be used for detailed studies of combined reconstruction perfomance, dead material, alignment with respect to the Inner Detector.

- The ATLAS Muon Spectrometer is alive and ready for beam! 